TRANSACTIONS OF THE

AMERICAN MATHEMATICAL SOCIETY

Volume 357, Number 2, Pages 795-823

S 0002-9947(04)03601-3

Article electronically published on September 23, 2004

\title{
THE ARONSSON-EULER EQUATION FOR ABSOLUTELY MINIMIZING LIPSCHITZ EXTENSIONS WITH RESPECT TO CARNOT-CARATHÉODORY METRICS
}

\author{
THOMAS BIESKE AND LUCA CAPOGNA
}

\begin{abstract}
We derive the Euler-Lagrange equation (also known in this setting as the Aronsson-Euler equation) for absolute minimizers of the $L^{\infty}$ variational problem

$$
\left\{\begin{array}{l}
\inf \left\|\nabla_{0} u\right\|_{L^{\infty}(\Omega)}, \\
u=g \in \operatorname{Lip}(\partial \Omega) \text { on } \partial \Omega,
\end{array}\right.
$$

where $\Omega \subset \mathbf{G}$ is an open subset of a Carnot group, $\nabla_{0} u$ denotes the horizontal gradient of $u: \Omega \rightarrow \mathbb{R}$, and the Lipschitz class is defined in relation to the Carnot-Carathéodory metric. In particular, we show that absolute minimizers are infinite harmonic in the viscosity sense. As a corollary we obtain the uniqueness of absolute minimizers in a large class of groups. This result extends previous work of Jensen and of Crandall, Evans and Gariepy. We also derive the Aronsson-Euler equation for more "regular" absolutely minimizing Lipschitz extensions corresponding to those Carnot-Carathéodory metrics which are associated to "free" systems of vector fields.
\end{abstract}

\section{INTRODUCTION}

In 1967 Aronsson began a systematic study of minimal Lipschitz extensions in Euclidean space. Such extensions are functions defined in a set $\Omega \subset \mathbb{R}^{n}$ with the property that

$$
\|u\|_{W^{1, \infty}(\Omega)} \leq\|w\|_{W^{1, \infty}(\Omega)}, \text { for all } w \text { such that } u-w \in W_{0}^{1, \infty}(\Omega) .
$$

If $\partial \Omega$ sufficiently smooth, then the restriction of $u$ to $\partial \Omega$ is a Lipschitz function and $u$ is its minimal Lipschitz extension. Such notion is very useful as a model for a large class of $L^{\infty}$ variational problems which arise in applications to engineering. However, in general minimal Lipschitz extensions are neither smooth nor unique. In 1], Aronsson introduced what may be considered as a canonical minimal Lipschitz extension, one which is minimal in every subset of $\Omega$. Such minimizers are called absolutely minimizing Lipschitz extension, or absolute minimizers for short. In 1], Aronsson showed that sufficiently smooth absolute minimizers are classical solutions of the Euler-Lagrange equation associated to this variational problem, the

Received by the editors November 11, 2002 and, in revised form, October 15, 2003.

2000 Mathematics Subject Classification. Primary 35H20, 53C17.

Key words and phrases. Absolute minimizers, sub-Riemannian geometry.

The second author was partially supported by NSF CAREER grant No. DMS-0134318. 
$\infty$-Laplacian equation

$$
\sum_{i, j=1}^{n} \partial_{x_{i}} \partial_{x_{j}} u \partial_{x_{i}} u \partial_{x_{j}} u=0 .
$$

(In deference to Aronsson, this equation has subsequently been referred to also as Aronsson's Euler equation, or AEE.) Aronsson studied classical solutions of (1.1) in [2, in particular proving their uniqueness. As a consequence one obtains the uniqueness of $C^{2}$ absolute minimizers. However, still thanks to Aronsson's results, it is rather easy to verify that in general there are no classical solutions (see [26, page 55). In 1993, in his seminal work [26, Jensen proved that absolute minimizers are solutions of (1.1) in the viscosity sense, and then established a uniqueness theorem for such viscosity solutions, thus obtaining the uniqueness of absolute minimizers. In 2000, Crandall, Evans and Gariepy [12], gave an alternative (and very elegant) argument for the derivation of Aronsson's Euler equation, using a rather different set of ideas from the one in 26]. Their proof makes use of comparison with "cone" functions, which are solutions of the eikonal equation $|\nabla u|=1$. Thanks to Barron, Jensen and Wang's recent paper [3] and to Crandall's even more recent [11, the role of this Hamilton-Jacobi equation (and its generalizations) in the $L^{\infty}$ variational theory has become better understood and has assumed a central character. In these papers, the authors study absolute minimizers for the variational problems

$$
\inf \|f(x, u, \nabla u)\|_{L^{\infty}(\Omega)},
$$

with some minimal hypothesis on $f(x, s, p)$ (e.g., quasiconvexity in $p$ ).

The definition of absolutely minimizing Lipschitz extensions can be naturally formulated in a metric space, as it does not require a differentiable structure. However, in contrast with the Euclidean picture, very little is known in this general setting. The study of classical Euclidean problems in PDE, harmonic analysis and potential theory in the context of suitably regular metric spaces endowed with a doubling Borel measure is one of the most exciting and challenging trends in modern analysis (see for instance [9], 24], 23, 28 and references therein).

In [28, Juutinen has proved that absolute minimizers exist in every separable length space. There are no uniqueness results yet in this general setting, and it seems very difficult to follow Jensen and Aronsson's approach, i.e. to study the Aronsson Euler equation, since the latter is not in divergence form and it is not obvious how to formulate it in a metric space (where, roughly speaking, one cannot use second derivatives).

In the present paper we study the derivation of the Aronsson Euler equation, and the question of uniqueness of absolute minimizers in the setting of Carnot groups. This is a large class of metric spaces 11 in which a rich algebraic structure has allowed for the development of a broad range of results in harmonic analysis and PDE (for an account of such results, see for instance [33] and [19]).

A Carnot group (or nilpotent stratified Lie group) is an analytic simply connected Lie group $\boldsymbol{G}$ whose Lie algebra $\mathfrak{g}$ is nilpotent and has a stratification $\mathfrak{g}=V^{1} \oplus \ldots \oplus$ $V^{r}$, with $\left[V^{1}, V^{j}\right]=V^{1+j}, j=1, \ldots, r-1$, and $\left[V^{j}, V^{r}\right]=0$ for $j=1, \ldots, r$. We let $X_{i, k}, k=1, \ldots, r$ and $i=1, \ldots, m_{k}$ with $m_{k}=\operatorname{dim}\left(V^{k}\right)$, denote a basis of $V^{k}$, and

\footnotetext{
${ }^{1}$ This class includes Euclidean space, but in general Carnot groups are not even locally biLipschitz equivalent to a Euclidean space.

${ }^{2}$ For simplicity, in the sequel we will denote $m_{1}$ by $m$.
} 
let $|\cdot|$ be a norm in $\mathfrak{g}$ for which the $X_{j, k}$ are orthonormal. Since the exponential map exp : $\mathfrak{g} \rightarrow \boldsymbol{G}$ is a diffeomorphism, then we can identify $\exp \left(\sum_{k} \sum_{i} x_{i, k} X_{i, k}\right)$ with the set of coordinates $x=\left\{x_{i, k}\right\}$. We denote by $d_{C}$ the Carnot-Carathéodory distance associated to the basis $X_{i}=X_{i, 1}, i=1, \ldots, m_{1}$, of the Lie algebra [33]: Let $x, y \in \boldsymbol{G}$, and for every $\delta>0$ define

$$
\begin{aligned}
A(\delta)=\{\gamma:[0, \delta] \rightarrow \boldsymbol{G} \mid \gamma(0)=x, \gamma(\delta)=y \\
\left.\quad \text { and } \gamma^{\prime}(s)=\sum_{i=1}^{m} a_{i}(s) X_{i}(\gamma(s)), \text { with } \sum_{i=1}^{m}\left|a_{i}\right| \leq 1\right\} .
\end{aligned}
$$

By virtue of Chow's theorem [10, we know that for $\delta$ large enough the set $A$ is nonempty. Let us define

$$
d_{C}(x, y)=\inf _{A(\delta)} \delta \text { not empty } \delta
$$

If $x \in \boldsymbol{G}$ and $r>0$, we will denote by $B_{C}(x, r)=\left\{y \in \boldsymbol{G} \mid d_{C}(x, y)<r\right\}$ the metric balls in the control metric $d_{C}$. We will also denote by $d(\cdot, \cdot)$ the pseudo-metric given by $d(x, y)=\left\|y^{-1} x\right\|$, where

$$
\|x\|^{2 r !}=\sum_{k=1}^{r}\left(\sum_{i=1}^{m_{k}}\left|x_{i, k}\right|^{2}\right)^{\frac{r !}{k}} .
$$

The two functions $d, d_{C}$ are equivalent, in the sense that there exists $C>0$ such that for any $x \in \boldsymbol{G}$ and $r>0$, one has $B\left(x, C^{-1} r\right) \subset B_{C}(x, r) \subset B(x, C r)$. The couple $\left(\boldsymbol{G}, d_{C}\right)$ is a separable length space (i.e., any two points can be joined by a length-minimizing curve) 31.

We will denote by $\nabla_{0}$ the horizontal gradient, i.e. the gradient along the horizontal directions $X_{1}, \ldots, X_{m_{1}}$. The notation $\nabla u$ will be used for the Euclidean gradient in the $x_{i, l}$ variables. Hereafter, whenever we write $u \in C^{2}$ we intend that the second derivatives $\partial_{x_{i, l}} \partial_{x_{i, k}} u$ are continuous, whereas for $k \in \mathbb{N}$, the symbol $u \in \Gamma^{k}$ implies that all the horizontal derivatives $X_{i_{1}} X_{i_{2}} \cdots X_{i_{k}} u$ of order $k$ are continuous.

Carnot groups were introduced by Folland in [16] (see also Folland and Stein [17] and Stein [33]). Such spaces arise naturally as ideal boundaries of noncompact rank one symmetric spaces. A well known instance is the Heisenberg group $\mathbb{H}^{n}, n \geq 1$ (see [17]), which is isomorphic to the nilpotent part of the Iwasawa decomposition of $U(n, 1)$, the isometry group of the complex hyperbolic space of dimension $n$. Such a group has a Lie algebra stratification $\mathfrak{h}=\mathbb{C}^{n} \times \mathbb{R}$, and group law $(z, t)\left(z^{\prime}, t^{\prime}\right)=$ $\left(z+z^{\prime}, t+t^{\prime}+2 \operatorname{Im}\left(\left\langle z, \bar{z}^{\prime}\right\rangle\right)\right.$. Carnot groups also arise as limits of Riemannian manifolds and as tangent spaces to Carnot-Carathéodory spaces (see [22], [30], [19] and $[20]$ ).

If $\Omega \subset \boldsymbol{G}$ is an open set and $u: \boldsymbol{G} \rightarrow \mathbb{R}$, then the Lipschitz norm of $u$ in $\Omega$ is given by

$$
\|u\|_{L i p(\Omega)}=\sup _{x, y \in \Omega, x \neq y} \frac{|u(x)-u(y)|}{d_{C}(x, y)} .
$$

Garofalo and Nhieu 21] have proved that $u$ is Lipschitz if and only if $u$ is weakly differentiable along the horizontal directions $X_{i}$ and $\left\|\nabla_{0} u\right\|_{L^{\infty}(\Omega)}<\infty$ (see also Franchi, Serapioni and Serra-Cassano [18] for an alternative proof of one of the two implications). Moreover,

$$
\|u\|_{L i p(\Omega)}=\left\|\nabla_{0} u\right\|_{L^{\infty}(\Omega)} .
$$


Definition 1.1. A function $u \in \operatorname{Lip}(\bar{\Omega})$ is an absolutely minimizing Lipschitz extension (of its trace) if for every $V \subset \Omega$ and $v \in \operatorname{Lip}(V)$ such that $u=v$ on $\partial V$, we have

$$
\left\|\nabla_{0} u\right\|_{L^{\infty}(V)} \leq\left\|\nabla_{0} v\right\|_{L^{\infty}(V)} .
$$

We now turn our attention to the Aronsson-Euler equation of the functional $\left\|\nabla_{0} u\right\|_{L^{\infty}}$, the subelliptic $\infty$-Laplacian equation

$$
\mathcal{L}_{\infty} u:=\sum_{i, j=1}^{m} X_{i} X_{j} u X_{i} u X_{j} u=0 .
$$

This is a fully non-linear, degenerate elliptic equation.

Definition 1.2. Let $\Omega \subset G$ be an open set. A function $u \in C(\Omega)$ is a viscosity subsolution of (1.5) in $\Omega$ if for every $x_{0} \in \Omega$ and every $\Gamma^{2}$ function $\phi$ defined in a neighborhood $U$ of $x_{0}$, such that $u\left(x_{0}\right)=\phi\left(x_{0}\right)$, and $u(x) \leq \phi(x)$ for $x \in U$, one has that $\mathcal{L}_{\infty} \phi\left(x_{0}\right) \geq 0$. We say that $u$ is a viscosity supersolution at $x_{0}$ if $-u$ is a viscosity subsolution at $x_{0}$, and finally that $u$ is a viscosity solution if it is both a subsolution and a supersolution in the viscosity sense.

We remark here that if $\phi$ is $\Gamma^{2}$, then both $X_{l} X_{i} \phi$ for $l, i=1, \ldots, m$ and $\partial_{x_{j, 2}} \phi$, for $j=1, \ldots, m_{2}$ are continuous functions.

Viscosity solutions of (1.5) have been recently studied in the papers [5, [13, [14] and [29]. We mention here that one could consider a stronger definition of (viscosity) solution, by assuming only that $u(x) \leq \phi(x)$ for $x \in H\left(x_{0}\right) \cap U$, where $H\left(x_{0}\right)=\operatorname{span}\left(X_{1}, \ldots, X_{m}\right)\left(x_{0}\right)$ is the so-called horizontal space at $x_{0}$. This definition emerges with respect to the Monge-Ampère equation in Carnot groups and to the definition of horizontal convexity introduced years ago by Caffarelli (and independently rediscovered in [13). In this paper we will only use definition 1.2 and our main motivation comes from the following theorem by Bieske.

Theorem 1.3 (Uniqueness of solutions of (1.5), 5]). Let $\Omega \subset \mathbb{H}^{n}$ be a connected open set, and $g \in C(\partial \Omega)$. There exists a unique viscosity solution (in the sense of Definition 1.2) of the Dirichlet problem

$$
\begin{cases}\mathcal{L}_{\infty} u=0 & \text { in } \Omega, \\ u=g & \text { in } \partial \Omega .\end{cases}
$$

The main result of the present paper is the following

Theorem 1.4 (Derivation of the Aronsson-Euler equation). If $u \in \operatorname{Lip}(\Omega)$ is an absolutely minimizing Lipschitz extension, then $u$ is a viscosity solution of (1.5) in $\Omega$.

In view of the existence theorem for absolute minimizers in [28, as an immediate corollary of Theorem 1.4 we have

Corollary 1.5 (Uniqueness of absolutely minimizing Lipschitz extensions). Given $\Omega \subset \mathbb{H}^{n}$, and $g \in \operatorname{Lip}(\partial \Omega)$, there exists a unique absolutely minimizing Lipschitz extension of $g$ in $\Omega$.

We point out that Theorem 1.4 cannot be obtained as a consequence of the results in [3] and [11], as the functional $\left|\nabla_{0} u\right|^{2}=f(x, \nabla u)$ is not quasiconvex in the $\nabla u$ component. This can be easily checked by a direct computation in the Heisenberg group setting. 
The proof of Theorem 1.4 is based on a comparison of the absolute minimizer $u$ with a suitably chosen test function $V$. This approach is inspired by the ideas in [12] and [11]. The argument of Crandall [11], in particular, provides the blueprint for our proof. The main difference between Crandall's proof and ours is in the construction of the test function. In [11] the commutation of mixed derivatives is used in a crucial way when differentiating the Hamilton-Jacobi equation. However in our setting the vector fields do not commute, resulting in the appearance of an extra term. This extra term affects the construction of the comparison function. In the proof of Theorem 1.4 we deal with this problem through a refinement of Crandall's original argument.

There are a number of immediate generalizations of Theorem 1.4 to more general functionals $f(x, s, p)$, where $p$ now denotes the variables corresponding to the horizontal gradient, in which $f(x, s, p)$ is required to satisfy conditions similar to (f1)-(f3) in [11. We will not address these generalizations here, and rather focus on the model case. A less obvious extension of our results concerns the derivation of the Aronsson Euler equation for absolute minimizers with respect to the Carnot-Carathéodory distance associated to more general systems of vector fields (as in 25). The reason why our proof does not extend to this setting is that we use in a crucial way the Taylor formula [17] and the approximation through group mollifiers. Another fundamental aspect of our proofs is the possibility of finding a "good" system of coordinates. While one can think of several ways to deal with the absence of either of these useful tools in the general setting (see for instance 8], [21], and [18]), it is not yet clear to us how to carry out the extension.

With the extra assumption that the horizontal derivatives of the absolute minimizer are continuous, in Section 4 we derive the Aronsson Euler equation for absolutely minimizing Lipschitz extensions with respect to the Carnot-Carathéodory metric associated to a system of smooth vector fields $X_{1}, \ldots, X_{m}$ which satisfy Hörmander's finite rank condition, i.e., at every point $x_{0} \in \mathbb{R}^{n}$, the set of all $X_{i}$ 's and their commutators up to order $r \in \mathbb{N}$ generates an $n$-dimensional vector space. If $X_{1}, \ldots, X_{m}$ satisfy Hörmander's condition, we will call them Hörmander vector fields. We also need to assume that the vector fields are free up to order $r$ (see Definition 4.2 in Section 4), in order to find an appropriate system of local coordinates. Our main tool in the proof of this result will be the approximation techniques developed by Rothschild and Stein [32]. In that fundamental paper, the authors showed how to approximate locally the analytic and geometric structure associated to a system of free Hörmander vector fields with a Carnot group structure (see Theorem 4.5). In the approximation there are error terms which are "well-behaved", and most of our proofs will deal with estimates of these error terms.

In many examples, one can construct adequate Taylor approximations for a given set of smooth Hörmander vector fields, thus avoiding the need to invoke the Carnot group approximation. In the last section of the paper we show a specific example in which the Rothschild-Stein theorem is not needed. For a larger, and more significative class of examples see [7], where non-free vector fields are studied 3

\footnotetext{
${ }^{3}$ After this paper was submitted, C. Wang informed us that he has proved a more general version of our result in his recent preprint [34. Namely, absolutely minimizing Lipschitz extensions with respect to any CC metric (associated to a system of Hörmander vector fields) are viscosity solutions of the infinite sub-Laplacian. His proof differs from ours in two points: (i) The comparison between the AMLE and the test function in his paper is based on the Euclidean Taylor
} 


\section{Preliminary Definitions AND RESUlts}

In this section we recall some basic definitions and results about Taylor polynomials in nilpotent Lie groups (see [17] for a more detailed treatment). We also provide an elementary proof of Theorem 1.4 for $C^{2}$ absolute minimizers, inspired by an argument by Jensen.

One of the most important features of Carnot groups is the existence of a oneparameter family of non-isotropic dilations $\delta_{s}(x)=\left(s^{k} x_{i, k}\right)$, for $s>0$. A function $f$ defined in $G$ is homogeneous of order $\lambda \in \mathbb{R}$ if $f\left(\delta_{s}(x)\right)=s^{\lambda} f(x)$ for any $s>0$. The elements of the basis $X_{i, k}$ act as $k$-th order differential operators when viewed as left invariant vector fields on $G$, i.e., $X_{i, k} f\left(\delta_{s}(x)\right)=s^{k}\left(X_{i, k} f\right)\left(\delta_{s}(x)\right)$.

Definition 2.1. A polynomial in $G$ is a function which can be expressed in the exponential coordinates as

$$
P(x)=\sum_{I} a_{I} x^{I},
$$

where $I=\left(i_{j, k}\right)_{j=1, \ldots, m_{k}}^{k=1, \ldots, r}$ is a multi-index, the $a_{I}$ are real numbers, and

$$
x^{I}=\prod_{\substack{k=1, \ldots, r ; \\ j=1, \ldots, m_{k}}} x_{j, k}^{i_{j, k}} .
$$

The homogeneous degree of the monomial $x^{I}$ is given by the sum

$$
\sum_{k=1}^{r} \sum_{j=1}^{m_{k}} k i_{j, k}
$$

The (homogeneous) degree of polynomials is defined accordingly. For any integer $d$, let us denote by $\mathcal{P}_{d}$ the set of polynomials of homogeneous degree less than or equal to $d$.

Next we need to express the vector fields $X_{i, k}$ in exponential coordinates. From the Baker-Campbell-Hausdorff formula we obtain, for $l=1, \ldots, r-1$ and $n=$ $1, \ldots, m_{l}$,

$$
X_{n, l}(x)=\partial_{x_{n, l}}+\sum_{\nu=l}^{r-1} \sum_{j=1}^{m_{\nu+1}} f_{j, \nu+1}^{n, l}\left(x_{1}, x_{2}, \ldots, x_{\nu+1-l}\right) \partial_{x_{j, \nu+1}},
$$

where $x_{i}=\left(x_{1, i}, \ldots, x_{m_{i}, i}\right)$, the $f_{j, k}^{n, l}$ are polynomials of degree $d=k-l$, and the components of $x_{\nu}$ may appear raised to the highest power of $\frac{k-l}{\nu}$ (the polynomial coefficients depend on the group constants). For $l=r$ we simply have $X_{n, r}(x)=$ $\partial_{x_{n, r}}$. From (2.1) it is immediate that if $P$ is a group polynomial of degree $d \in \mathbb{N}$, then $X_{i, 1} P$ is a group polynomial of degree $d-1$. If $d \geq k$, then $X_{i, k} P$ is a polynomial of degree $d-k$.

Recall that if we consider a multi-index $I=\left[\left(i_{1}, k_{1}\right),\left(i_{2}, k_{2}\right), \ldots,\left(i_{l}, k_{l}\right)\right]$, with $l \in \mathbb{N}, 0 \leq k_{j} \leq r$, and $1 \leq i_{j} \leq m_{k_{j}}$, then if $f$ is a smooth function defined in $G$, we will denote a derivative of $f$ of order $|I|=\sum_{j=1}^{l} k_{j}$ by

$$
X^{I} f=X_{i_{1}, k_{1}} X_{i_{2}, k_{2}} \ldots X_{i_{l}, k_{l}} f .
$$

polynomials (by a clever adaptation of Crandall's argument) and not on the group Taylor polynomials. (ii) Once the test function is constructed, he uses the argument in [3] rather than follow Crandall's paper [11. On the other hand, our construction of explicit test functions with controlled "subelliptic" Taylor polynomials may be useful in studying more general fully non-linear equations. 
Next we recall the definition of (left) Taylor polynomial in Carnot groups (see pg. 35 , in [17]).

Definition 2.2. If $f \in C_{0}^{\infty}(G)$ and $k$ is an integer, then the $k$-th order Taylor polynomial $P_{k}$ of $f$ at the origin 0 is the unique polynomial of homogeneous order less than or equal to $k$ such that

$$
X^{I} P_{k}(0)=X^{I} f(0),
$$

for all multi-indices $I$ such that $|I| \leq k$.

The following theorem is the stratified Taylor inequality (see [17, Theorem 1.42).

Theorem 2.3. If $k \geq 1$, then there exist positive constants $C_{k}$ and $b$ such that for all $u \in \Gamma^{k}(\boldsymbol{G})$, and for all $x, y \in \boldsymbol{G}$,

$$
\left|u(x y)-P_{k}(y)\right| \leq C_{k}|| y \|^{k} \sup _{B\left(0, b^{k}|| y||\right),|I|=k}\left|X^{I} u(x z)-X^{I} u(x)\right| .
$$

Here $P_{k}(y)$ denotes the $k$-th order Taylor polynomial of $u$ at the point $x$.

It is useful to write the polynomial $P_{2}$ explicitly for a function $u \in \Gamma^{2}(\boldsymbol{G})$ at the origin: Set $T=\left(\partial_{x_{1,2}} u(0), \ldots, \partial_{x_{m_{2}, 2}} u(0)\right), x_{1}=\left(x_{1,1}, \ldots, x_{m, 1}\right), x_{2}=\left(x_{1,2}, \ldots, x_{m_{2}, 2}\right)$ and denote by

$$
H=\left(\begin{array}{llll}
X_{1} X_{1} u(0), & \ldots & , & X_{1} X_{m} u(0) \\
\ldots & & \ldots \\
X_{m} X_{1} u(0), & \ldots & , & X_{m} X_{m} u(0)
\end{array}\right)
$$

the horizontal Hessian of $u$ at the origin, and by

$$
H^{\star}=\frac{1}{2}\left(H+H^{T}\right)
$$

the symmetrized horizontal Hessian. With this notation we have

$$
\begin{aligned}
P_{2}(x) & =u(0)+\left\langle\nabla_{0} u(0), x_{1}\right\rangle+\left\langle T, x_{2}\right\rangle+\frac{1}{2}\langle H x, x\rangle \\
& =u(0)+\left\langle\nabla_{0} u(0), x_{1}\right\rangle+\left\langle T, x_{2}\right\rangle+\frac{1}{2}\left\langle H^{\star} x, x\right\rangle .
\end{aligned}
$$

We will need the following elementary consequence of Theorem 2.3 .

Corollary 2.4. Let $U \subset \boldsymbol{G}$ be a neighborhood of the origin, $k \in \mathbb{N}, k \geq 2$, and $u, v \in$ $\Gamma^{k}(U)$. Denote by $H^{\star}$ and $K^{\star}$ the symmetrized Hessians of $u$ and $v$ respectively. If $u(0)=v(0)$ and $\partial_{x_{i, k}} u(0)=\partial_{x_{i, k}} v(0)$, for $k=1,2$ and $i=1, \ldots, m_{k}$, and if $H^{\star}>K^{\star}$, then there exists $\epsilon=\epsilon(u, v, \boldsymbol{G})>0$ such that $u>v$ in $B(0, \epsilon) \backslash\{0\} \subset U$.

We conclude this section with a simple proof of the derivation of the Aronsson Euler equation for $\Gamma^{2}$ absolutely minimizing Lipschitz extensions in Carnot groups.

Theorem 2.5. Let $\Omega \subset G$ be an open domain. If $u \in \Gamma^{2}(\Omega)$ is an absolutely minimizing Lipschitz extension, then $u$ is a (classical) solution of (1.5) in $\Omega$.

Proof. Let $x^{\prime}$ be an arbitrary point in the domain $\Omega$, which in turn is a subset of the Carnot group $\boldsymbol{G}$, and let $u$ be a $\Gamma^{2}$ absolute minimizer. We wish to show that $u$ is infinite harmonic at $x^{\prime}$. Since the $\infty$-Laplacian operator $\mathcal{L}_{\infty}$ is left-invariant, then without loss of generality we may assume $x^{\prime}$ is the origin. By the smoothness 
of $u$, we may use the second order Taylor polynomial formula given by equation (2.4) and the error estimates in Theorem 2.3 to obtain

$$
\begin{aligned}
u(p) & =u(0)+\left\langle\nabla_{0} u(0), x_{1}\right\rangle+\left\langle T, x_{2}\right\rangle+\frac{1}{2}\left\langle H^{\star} x_{1}, x_{1}\right\rangle+o\left(\|p\|^{2}\right) \\
& =u(0)+\sum_{i=1}^{m} x_{i, 1} X_{i} u(0)+\sum_{i=1}^{m_{2}} x_{i, 2} X_{i, 2} u(0)+\frac{1}{2} \sum_{i, j=1}^{m} H_{i j}^{\star} x_{i, 1} x_{j, 1}+o\left(\|p\|^{2}\right) .
\end{aligned}
$$

Using this expansion, we obtain, for any $k \in\{1,2, \ldots, m\}$,

$$
X_{k} u(p)=X_{k} u(0)+\sum_{i=1}^{m} H_{i k}^{\star} x_{i, 1}+\sum_{j=1}^{m_{2}} f_{j, 2}^{k, 1}\left(x_{1}\right) X_{j, 2} u(0)+o(\|p\|),
$$

where the $f_{j, 2}^{k, 1}$ are as in equation (2.1). Recall that the various $f_{j, 2}^{k, 1}$ are polynomials of homogeneous degree one, and in particular, we have

$$
f_{j, 2}^{k, 1}=\sum_{i=1}^{m} c_{k, i}^{j} x_{i, 1},
$$

where the antisymmetry of the Lie bracket operation produces

$$
c_{k, i}^{j}=-c_{i, k}^{j} \text {. }
$$

Squaring (2.5), we arrive at

$$
\begin{aligned}
\left(X_{k} u(p)\right)^{2}= & \left(X_{k} u(0)\right)^{2}+2 X_{k} u(0) \sum_{i=1}^{m} H_{i k}^{\star} x_{i, 1} \\
& +2 X_{k} u(0) \sum_{j=1}^{m_{2}} f_{j, 2}^{k, 1}\left(x_{1}\right) X_{j, 2} u(0)+o(\|p\|) .
\end{aligned}
$$

The latter, combined with a standard computation, produces

$$
\left\|\nabla_{0} u(p)\right\|^{2}=\left\|\nabla_{0} u(0)\right\|^{2}+2 \sum_{i=1}^{m} P_{i} x_{i, 1}+o(\|p\|),
$$

where the coefficient $P_{i}$ is given by

$$
P_{i}=\sum_{k=1}^{m} X_{k} u(0) H_{i k}^{\star}+\sum_{k=1}^{m} \sum_{j=1}^{m_{2}} c_{k, i}^{j} X_{k} u(0) X_{j, 2} u(0) .
$$

If $P_{i}=0, i=1, \ldots, m$, then clearly $\sum_{i=1}^{m} P_{i} X_{i} u(0)=0$, and hence in view of (2.6) we would have

$$
\begin{aligned}
\mathcal{L}_{\infty} u(0) & =\sum_{i=1}^{m} \sum_{k=1}^{m} X_{k} u(0) X_{i} u(0) H_{i k}^{\star} \\
& =-\sum_{i=1}^{m} \sum_{k=1}^{m} \sum_{j=1}^{m_{2}} c_{k, i}^{j} X_{i} u(0) X_{k} u(0) X_{j, 2} u(0)=0,
\end{aligned}
$$

thus concluding the proof of the theorem.

We are left with the case

$$
\sum_{i=1}^{m} P_{i}^{2} \neq 0
$$


Note that the maximum of $\left\|\nabla_{0} u(p)\right\|^{2}$ in the Euclidean ball $B_{E}(0, \eta)=\{p$ : $\left.\sum_{i, j} x_{i, j}^{2} \leq \eta^{2}\right\}$ occurs when $\sum_{i=1}^{m} x_{i, 1}^{2}=\eta^{2}$, and in particular when

$$
x_{i, 1}=\eta P_{i}\left(\sum_{j=1}^{m} P_{j}^{2}\right)^{-\frac{1}{2}}
$$

We thus conclude that

$$
\left\|\nabla_{0} u(p)\right\|_{L^{\infty}\left(B_{E}(0, \eta)\right)}^{2}=\left\|\nabla_{0} u(0)\right\|^{2}+2 \eta\left(\sum_{i=1}^{m} P_{i}^{2}\right)^{\frac{1}{2}}+o(\eta) .
$$

Next, we consider the $\Gamma^{2}$ function $v$ defined by

$$
v(p)=u(p)+\frac{\gamma}{2}\left(\varepsilon^{2}-\sum_{k=1}^{r} \sum_{i=1}^{m_{k}} x_{i, k}^{2}\right),
$$

where $\gamma$ and $\varepsilon$ are parameters sufficiently close to 0 . Proceeding as above, we compute the Taylor polynomial for $v$ as

$$
\begin{aligned}
v(p)= & u(0)+\frac{\gamma}{2} \varepsilon^{2}+\sum_{i=1}^{m} x_{i, 1} X_{i} u(0)+\sum_{i=1}^{m_{2}} x_{i, 2} X_{i, 2} u(0) \\
& +\frac{1}{2} \sum_{i, j=1}^{m} \widetilde{H^{\star}}{ }_{i j} x_{i, 1} x_{j, 1}+o\left(\|p\|^{2}\right),
\end{aligned}
$$

where $\widetilde{H}^{\star}{ }_{i j}=H_{i j}^{\star}-\gamma \delta_{i j}$. Observing that $u=v$ on the Euclidean sphere of radius $\varepsilon$, we proceed as above and conclude that

$$
\begin{aligned}
\left\|\nabla_{0} u(p)\right\|_{L^{\infty}\left(B_{E}(0, \varepsilon)\right)}^{2} & =\left\|\nabla_{0} u(0)\right\|^{2}+2 \varepsilon\left(\sum_{i=1}^{m} P_{i}^{2}\right)^{\frac{1}{2}}+o(\varepsilon), \\
\left\|\nabla_{0} v(p)\right\|_{L^{\infty}\left(B_{E}(0, \varepsilon)\right)}^{2} & =\left\|\nabla_{0} u(0)\right\|^{2}+2 \varepsilon\left(\sum_{i=1}^{m} \tilde{P}_{i}^{2}\right)^{\frac{1}{2}}+o(\varepsilon),
\end{aligned}
$$

where $P_{i}$ is defined as above and $\tilde{P}_{i}$ is defined analogously,

$$
\tilde{P}_{i}=\sum_{k=1}^{m} X_{k} u(0){\widetilde{H^{\star}}}_{i k}+\sum_{k=1}^{m} \sum_{j=1}^{m_{2}} c_{k, i}^{j} X_{k} u(0) X_{j, 2} u(0)=P_{i}-\gamma X_{i} u(0) .
$$

Since $u$ is an absolute minimizer, we have

$$
0 \leq\left\|\nabla_{0} v(p)\right\|_{L_{\infty}\left(B_{E}(0, \varepsilon)\right)}^{2}-\left\|\nabla_{0} u(p)\right\|_{L_{\infty}\left(B_{E}(0, \varepsilon)\right)}^{2} .
$$

That is,

$$
0 \leq 2 \varepsilon\left(\left(\sum_{i=1}^{m} \tilde{P}_{i}^{2}\right)^{\frac{1}{2}}-\left(\sum_{i=1}^{m} P_{i}^{2}\right)^{\frac{1}{2}}\right)+o(\varepsilon),
$$

so that, dividing by $2 \varepsilon$ and letting $\varepsilon \rightarrow 0$, we obtain

$$
0 \leq\left(\sum_{i=1}^{m} \tilde{P}_{i}^{2}\right)^{\frac{1}{2}}-\left(\sum_{i=1}^{m} P_{i}^{2}\right)^{\frac{1}{2}}
$$

and consequently,

$$
0 \leq \sum_{i=1}^{m} \tilde{P}_{i}^{2}-\sum_{i=1}^{m} P_{i}^{2}
$$


Using the definition of $\widetilde{H^{\star}}{ }_{i j}$, we compute

$$
\tilde{P}_{i}^{2}=P_{i}^{2}+2 \gamma P_{i} X_{i} u(0)+\left(\gamma X_{i} u(0)\right)^{2},
$$

so that we ultimately arrive at

$$
0 \leq \sum_{i=1}^{m}\left(2 \gamma P_{i} X_{i} u(0)+\left(\gamma X_{i} u(0)\right)^{2}\right) \equiv F(\gamma)
$$

Now $F(0)=0$, and so

$$
\frac{F(\gamma)-F(0)}{\gamma-0}=\sum_{i=1}^{m} P_{i} X_{i} u(0)+\gamma \sum_{i=1}^{m}\left(X_{i} u(0)\right)^{2},
$$

so that

$$
F^{\prime}(0)=\sum_{i=1}^{m} P_{i} X_{i} u(0)
$$

and we conclude that

$$
\begin{aligned}
0 & =\sum_{i=1}^{m} P_{i} X_{i} u(0) \\
& =\sum_{i=1}^{m} \sum_{k=1}^{m} X_{i} u(0) X_{k} u(0) H_{i k}^{\star}+\sum_{i=1}^{m} \sum_{k=1}^{m} \sum_{j=1}^{m_{2}} X_{i} u(0) X_{k} u(0) X_{j, 2} u(0) c_{k, i}^{j} \\
& =\mathcal{L}_{\infty} u(0)+0,
\end{aligned}
$$

where the last equality is a consequence of (2.6).

The previous argument is very similar to the proof of the corresponding Euclidean result in [26]. The main difference rests in the shape of the Taylor polynomial, which accounts for an extra term in the second order part $P_{i}$.

\section{Derivation of the Aronsson-Euler equations FOR LIPSCHITZ ABSOLUTE MINIMIZERS IN CARNOT GROUPS}

In this section we prove Theorem 1.4. We start with a lemma which extends Crandall's argument in [11], pages 5-6, to the Carnot group setting.

Lemma 3.1. Let $u \in \operatorname{Lip}(B(0,1)), u(0)=0$ and set $\mu>0$. If for $\epsilon>0$ small enough one can find a function $V_{\epsilon} \in \Gamma^{2}\left(\bar{U}_{\epsilon}\right)$, where $U_{\epsilon} \subset B\left(0, \sqrt{\frac{\epsilon}{\mu}}\right)$ is a neighborhood of the origin, such that

$$
\begin{aligned}
V_{\epsilon}(0) & =-\epsilon \\
V_{\epsilon} & <u \text { in } U_{\epsilon}, \\
V_{\epsilon} & =u \text { on } \partial U_{\epsilon}, \\
u & <V_{\epsilon} \text { outside the ball } B\left(0, \sqrt{\frac{\epsilon}{\mu}}\right)
\end{aligned}
$$

and

$$
\left|\nabla_{0} V_{\epsilon}\right|=1 \text { in a neighborhood of } U_{\epsilon},
$$

then $u$ cannot be absolutely minimizing in $B(0,1)$. 
Proof. We argue by contradiction. Assume that $u$ is an absolute minimizer; then

$$
\left|\nabla_{0} u\right| \leq 1,
$$

a.e. in $U_{\epsilon}$. Let $\gamma$ be the horizontal curve obtained as a solution of the ODE

$$
\frac{d}{d t} \gamma=-\left.\left[\left(\nabla_{0} V_{\epsilon}\right) \nabla_{0}\right]\right|_{\gamma}, \text { and } \gamma(0)=0
$$

Since $\left|\frac{d}{d t} \gamma\right|=1$, and $\gamma$ is horizontal, then there exist $\epsilon>0$ and $C_{\epsilon}>0$ such that for $0 \leq t<\epsilon$ one has

$$
C_{\epsilon}^{-1} t \leq\|\gamma\|_{G} \leq C_{\epsilon} t
$$

Let us assume at this point that $u \in \Gamma^{1}(B(0,1))$, and show how to conclude the proof with this extra hypothesis. A simple computation gives

$$
\frac{d}{d t}\left(V_{\epsilon}(\gamma)-u(\gamma)\right)=-\left\langle\nabla_{0} V_{\epsilon},\left(\nabla_{0} V_{\epsilon}(\gamma)-\nabla_{0} u(\gamma)\right)\right\rangle .
$$

From (3.3) we have

$$
\frac{d}{d t}\left(V_{\epsilon}(\gamma)-u(\gamma)\right) \leq 0
$$

Hence for any $t>0$,

$$
\left(V_{\epsilon}(\gamma)-u(\gamma)\right)(t) \leq\left(V_{\epsilon}(\gamma)-u(\gamma)\right)(0)=-\epsilon .
$$

The latter implies that $\gamma \in U_{\epsilon}$ for any $t>0$, but this contradicts (3.5) and the fact that $U_{\epsilon} \subset B\left(0, \sqrt{\frac{\epsilon}{\mu}}\right)$. In fact, the curve $\gamma$ will exit the ball $B\left(0, \sqrt{\frac{\epsilon}{\mu}}\right)$ after a time roughly equivalent to $\sqrt{\epsilon}$.

If $u \notin \Gamma^{1}(B(0,1))$, then (3.6) does not hold in general for every $t>0$. To circumvent this obstacle we mollify the function $u$. We emphasize that only at this point of the proof do we use the group structure.

Let $\phi \in C_{0}^{\infty}(B(0,2)), \phi \geq 0$ and $\int_{G} \phi d H=1$. For any $k \in \mathbb{N}$, set $\phi_{k}(x)=$ $k^{Q} \phi\left(\delta_{\frac{1}{k}}(x)\right)$ and

$$
u_{k}(x):=u * \phi_{k}=\int_{G} u\left(y^{-1} x\right) \phi_{k}(y) d H(y) .
$$

Since the horizontal vector fields $X_{1}, \ldots, X_{m}$ are left-invariant, then $\nabla_{0} u_{k}=\left(\nabla_{0} u\right) *$ $\phi_{k}$. Consequently

$$
\left\|\nabla_{0} u_{k}\right\|_{L^{\infty}\left(U_{\epsilon}\right)} \leq\left\|\nabla_{0} u\right\|_{L^{\infty}\left(U_{\epsilon}\right)} \leq 1
$$

For any $\epsilon, \delta>0$ we can find $k_{0}$ large enough so that

$$
\begin{aligned}
& u_{k}(0)>-\frac{\epsilon}{2}>V_{\epsilon}(0), \\
& u_{k} \leq V_{\epsilon}+\delta \text { outside the ball } B\left(0, \sqrt{\frac{\epsilon}{\mu}}\right)
\end{aligned}
$$

Repeating the argument in (3.6) and (3.7), we obtain

$$
V_{\epsilon}(\gamma)-u_{k}(\gamma) \leq V_{\epsilon}(0)-u_{k}(0)<-\frac{\epsilon}{2}
$$

hence for every $t>0$ we have

$$
V_{\epsilon}(\gamma)(t)<u_{k}(\gamma)(t)-\frac{\epsilon}{2}
$$


On the other hand, in view of (3.5), after a time roughly equal to $\sqrt{\epsilon}$ we have $\gamma(t) \notin B\left(0, \sqrt{\frac{\epsilon}{\mu}}\right)$. From (3.11) and (3.9) we have

$$
u_{k}(\gamma)-\delta \leq u_{k}(\gamma)-\frac{\epsilon}{2},
$$

after a time roughly equal to $\sqrt{\epsilon}$ has elapsed. If we choose $\delta<\frac{\epsilon}{2}$ and $k$ sufficiently large, we obtain a contradiction.

We are ready now to proceed with the proof of the main result.

Proof of Theorem 1.4. Let $u \in \operatorname{Lip}(\Omega)$ be an absolutely minimizing Lipschitz extension, and assume that there is a point where it fails to be $\infty$-harmonic in the viscosity sense. By left invariance of the vector fields (and of the equation) we can assume without loss of generality that such a point is the origin. Let $\phi$ be a $\Gamma^{2}$ function defined in a neighborhood of the origin such that

$$
\begin{aligned}
& u(0)=\phi(0), \\
& u(x) \leq \phi(x) \text { for every } x \text { in a neighborhood of the origin, } \\
& \mathcal{L}_{\infty} \phi(0)<0 .
\end{aligned}
$$

To simplify the notation, we let

$m \times m$ symmetric matrix $\quad H=\left\{H_{i j}\right\}$, with $H_{i j}=\frac{1}{2}\left(X_{i} X_{j} \phi(0)+X_{j} X_{i} \phi(0)\right)$,

$$
\begin{aligned}
m \text { vector } & D=\nabla_{0} \phi(0), \\
m \text { vector } & B=-H D, \\
m_{2} \text { vector } & T=\left\{\partial_{x_{i} 2} \phi(0)\right\} \text { for } i=1, \ldots, m_{2} .
\end{aligned}
$$

With this notation, (3.13) reads

$$
\langle B, D\rangle>0, \text { and }\langle H D, D\rangle<0 \text {. }
$$

Since the $\infty$-Laplacian $\mathcal{L}_{\infty}$ is a homogeneous operator of order 4 , which is invariant under the action of the orthogonal groups $O\left(m_{i}\right)$ acting on the spaces $V^{i}$, without loss of generality we can assume that

$$
D=(1,0, \ldots, 0) \in \mathbb{R}^{m} \text { and } T=(\tau, 0, \ldots, 0) \in \mathbb{R}^{m_{2}} .
$$

Denote by $C=\left\{C_{i j}\right\}$ the antisymmetric $m \times m$ matrix

$$
C_{j i}=\frac{1}{2}\left[X_{i}, X_{j}\right] \phi(0) .
$$

In the case of the Heisenberg group, $C$ is the symplectic matrix $J$. We now proceed with the construction of a comparison function $V_{\epsilon}$, with the property listed in the hypothesis of Lemma 3.1. The construction closely follows the argument in [3] and [11, but it is more delicate, as the vector fields do not commute and the Taylor formula reflects the anisotropicity of the metric.

We will build $V_{\epsilon}$ as a solution of a Hamilton-Jacobi equation. Our first step consists in constructing the initial data and the initial manifold.

Step 1. Let $M=P+H$, where $P$ is an $m \times m$ symmetric matrix defined by the formula

$$
P z=z+\frac{\langle(C-H) D, z\rangle}{\langle(C-H) D, D\rangle}(C-H) D-\langle D, z\rangle D, \text { for any } z \in \mathbb{R}^{m} .
$$


It is easy to verify the following properties of $M$ : for every $z, z^{\prime} \in \mathbb{R}^{m}$,

$$
\begin{aligned}
& \langle P z, z\rangle>0, \\
& \left\langle P z, z^{\prime}\right\rangle=\left\langle P z^{\prime}, z\right\rangle, \\
& P D=(C-H) D, \text { and so }\langle P D, D\rangle=-\langle H D, D\rangle>0 .
\end{aligned}
$$

Hence we have

$$
M>H \text { and } M D=C D .
$$

Step 2. Define the hyperplane

$$
L=\left\{x \in \mathfrak{g} \text { such that } \sum_{j=1}^{m} B_{j} x_{j, 1}=0\right\} .
$$

Note that for any $y_{i, k}, k=2, \ldots, r$ and $i=1, \ldots, m_{k}$, we have $\left(D, y_{i, k}\right) \notin L$. Let $N$ be the dimension of $\mathfrak{g}$. If we denote by $Y_{1}, \ldots, Y_{N-1}$ a basis of $L$, then $\left\{Y_{1}, \ldots, Y_{N-1}, D\right\}$ is a basis of $\mathfrak{g}=\mathbb{R}^{N}$. Set

$$
h(x)=\left\langle D, x_{1}\right\rangle+\frac{1}{2}\left\langle M x_{1}, x_{1}\right\rangle+\left\langle T, x_{2}\right\rangle,
$$

where we have set $x_{1}=\left(x_{1,1}, x_{2,1}, \ldots, x_{m, 1}\right)$ and $x_{2}=\left(x_{1,2}, \ldots, x_{m_{2}, 2}\right)$.

Step 3. We use the method of characteristics (see [15], 3.2.3 to 3.2.4) to solve the Cauchy problem for the eikonal equation

$$
\left\{\begin{array}{l}
\left|\nabla_{0} V\right|=1 \text { in a neighborhood } O \text { of the origin, } \\
V=h \text { on } L \cap O
\end{array}\right.
$$

subject to the constraint

$$
\partial_{x_{i, k}} V(0)=\partial_{x_{i, k}} \phi(0), \text { for } k=1,2 \text { and } i=1, \ldots, m_{k} .
$$

To obtain a local solution in a neighborhood of the origin, we need to verify that $L$ is not characteristic for 3.18 at the origin. If we write the vector fields as $X_{i}=\sum_{\alpha} a_{i \alpha} \partial_{x_{\alpha}}$ and set, for $p \in \mathfrak{g}$,

$$
f(x, p)=\frac{1}{2} \sum_{i=1}^{m}\left[a_{i \alpha} p_{\alpha}\right]^{2}
$$

and

$$
\psi(x)=\langle B, x\rangle,
$$

then the non-characteristic condition ([27]) is

$$
\left\langle\nabla_{p} f(x, p), \nabla_{x} \psi\right\rangle \neq 0
$$

when $x=0$ and $p=\nabla V(0)$. Here we have denoted by $\nabla_{p}$ and $\nabla_{x}$ the Euclidean derivatives in the $p$ and $x$ variables. Note that $f(0, \nabla \phi(0))=f\left(0, \partial_{x_{i, 1}} \phi(0), i=\right.$ $1, \ldots, m)=1 / 2$. A direct computation and the constraint (3.19) yield that at the origin one has

$$
\left\langle\nabla_{p} f(x, p), \nabla_{x} \psi\right\rangle=\sum_{i=1}^{m}\left(X_{i} V\right) X_{i} \psi=\langle B, D\rangle>0 .
$$

Step 4. We want to show that

$$
\mathcal{V}_{i j}=M_{i j}
$$


where $\mathcal{V}_{i j}=\frac{1}{2}\left(X_{i} X_{j} V(0)+X_{j} X_{i} V(0)\right)$. This is the part of the proof where the noncommutativity of the horizontal vector fields plays a major role. Let us differentiate the eikonal equation (3.18) along the direction $X_{i}$. We obtain

$$
\sum_{j=1}^{m} X_{i} X_{j} V X_{j} V=0
$$

When we evaluate the latter at the origin, we obtain

$$
\begin{aligned}
\mathcal{V}_{i j} D_{j} & =\sum_{j=1}^{m} \frac{1}{2}\left(X_{i} X_{j} V(0)+X_{j} X_{i} V(0)\right) D_{j}=\sum_{j=1}^{m} \frac{1}{2}\left[X_{j}, X_{i}\right] V(0) D_{j} \\
& =\sum_{j=1}^{m} C_{i j} D_{j}=(\text { by }(3.17) \text { and }(3.19))=\sum_{j=1}^{m} M_{i j} D_{j} .
\end{aligned}
$$

On the other hand, if $x, x^{\prime} \in L$, then the horizontal vectors

$$
Z=\sum_{i=1}^{m} x_{i, 1} X_{i} \text { and } Z^{\prime}=\sum_{i=1}^{m} x_{i, 1}^{\prime} X_{i}
$$

are tangent to $L$. Since $V=h$ along $L$, then

$$
\begin{aligned}
Z Z^{\prime} V=Z Z^{\prime} h & =\sum_{i, j=1}^{m} x_{i, 1} x_{j, 1}^{\prime} X_{i} X_{j} h \\
& =\sum_{i, j=1}^{m} x_{i, 1} x_{j, 1}^{\prime} X_{i} X_{j}\left(\frac{1}{2}\left\langle M x_{1}, x_{1}^{\prime}\right\rangle+\left\langle T, x_{2}\right\rangle\right) .
\end{aligned}
$$

An explicit computation yields

$$
\begin{aligned}
\left\langle\mathcal{V} x_{1}, x_{1}^{\prime}\right\rangle & =\frac{1}{2}\left(Z^{\prime} Z+Z Z^{\prime}\right) V(0) \\
& =\left\langle M x_{1}, x_{1}^{\prime}\right\rangle .
\end{aligned}
$$

Hence for any $x, x^{\prime} \in L$ we have

$$
\left\langle M x_{1}, x_{1}^{\prime}\right\rangle=\left\langle\mathcal{V} x_{1}, x_{1}^{\prime}\right\rangle
$$

Since for any $\xi \in \mathfrak{g}$ we can write $\xi=\sum_{i=1}^{N-1} y_{i} Y_{i}+d D$, then by virtue of (3.23), (3.26) and the symmetry of $M$, we obtain

$$
\left\langle M \xi_{1}, \xi_{1}\right\rangle=\left\langle\mathcal{V} \xi_{1}, \xi_{1}\right\rangle
$$

Equality (3.21) follows from the latter and from the polarization identity.

Step 5. From the results in Steps 1-4 we have that $V$ is a smooth function in a neighborhood of the origin such that

$$
V(0)=\phi(0)=0, \nabla_{0} V(0)=D,\left\{\partial_{x_{i, 2}} V(0)\right\}=T_{i}, i=1, \ldots, m_{2},
$$

and

$$
\mathcal{V}=M>H
$$

For $\epsilon>0$, set $V_{\epsilon}=V-\epsilon$. From Corollary 2.4 (essentially Taylor's theorem) we immediately obtain that

$$
V_{\epsilon}>\phi-\epsilon
$$


in $O \backslash\{0\}$, where $O$ is a neighborhood of the origin (independent of $\epsilon$ ). For $\mu>0$ sufficiently small, we can find a neighborhood of the origin $O_{\mu} \subset O$ such that

$$
V_{\epsilon}(x)>\phi(x)-\epsilon+\mu\|x\|^{2}, \text { for } x \in O_{\mu} \backslash\{0\} .
$$

Observe that for $\epsilon>0$ small enough, such that $B\left(0, \sqrt{\frac{\epsilon}{\mu}}\right) \subset O_{\mu}$, and for

$$
r \geq \sqrt{\frac{\epsilon}{\mu}} \text { and } x \in \partial B(0, r) \cap O_{\mu},
$$

one has

$$
u(x) \leq \phi(x) \leq \phi(x)+\left(\mu r^{2}-\epsilon\right)=\phi(x)-\epsilon+\mu\|x\|^{2}<V_{\epsilon}(x) .
$$

Hence there exist: 4 a neighborhood $U_{\epsilon}$ of the origin such that

$$
V_{\epsilon}=u \text { in } \partial U_{\epsilon} \text {, and } V_{\epsilon}<u \text { in } U_{\epsilon} .
$$

Without loss of generality we can assume that $U_{\epsilon}$ is connected. From (3.28) we have $U_{\epsilon} \subset B\left(0, \sqrt{\frac{\epsilon}{\mu}}\right)$. The contradiction now stems from (3.28), (3.29) and Lemma 3.1 .

\section{Derivation of the Aronsson-Euler equation FOR $\Gamma^{1}$ ABSOLUTE MINIMIZERS \\ WITH RESPECT TO MORE GENERAL CARNOT-CARATHÉOdORY METRICS}

In this section we study the Aronsson-Euler equation for absolutely minimizing Lipschitz extensions corresponding to those Carnot-Carathéodory metrics associated to systems of smooth vector fields which satisfy Hörmander's finite rank hypothesis (with rank $r \in \mathbb{N}$ ) and are free up to step $r$. In this case, we can approximate the structure of the CC space with an osculating Carnot group, using techniques and ideas which were first introduced by Rothschild and Stein in their celebrated paper [32].

We believe that a more direct proof could be given, without using this approximation scheme. In the last section of the paper we show how to derive the same theorem, in a particular example, without using the Rothschild-Stein results. Apart from the study of the Grushin case [7, the general non-free Hörmander case is still open.

Let $X_{1}, \ldots, X_{m}$ be a system of smooth vector fields in $\mathbb{R}^{n}$, which satisfy Hörmander's finite rank condition [25] up to step $r \in \mathbb{N}$, i.e., at every $x \in \mathbb{R}^{n}$, the vector fields and all their commutators up to order $r$ generate $\mathbb{R}^{n}$.

In this section we want to extend the results of Theorem 1.4. and derive the Aronsson-Euler equation for absolutely minimizing Lipschitz extensions with respect to the Carnot-Carathéodory metric associated to the system $X_{1}, \ldots, X_{m}$, in the presence of an extra assumption: the vector fields are free up to step $r$.

Definition 4.1. A free Lie algebra $\mathfrak{g}_{m, s}$ is a nilpotent Lie algebra of step $s$ having $m$ generators, but otherwise as few relations among the commutators as possible.

To construct such an object we first need to consider the infinite dimensional, free Lie algebra $G_{F}$ on $n$ generators $Y_{1}, \ldots, Y_{n}$. Roughly speaking, the only relations among the commutators of the $Y_{i}$ 's are given by anticommutativity and the Jacobi identity. For each $i \in \mathbb{N}$, define $G_{F}^{i}$ inductively as $G_{F}^{1}=G_{F}$, and $G_{F}^{i+1}=\left[G_{F}^{i}, G_{F}\right]$.

\footnotetext{
${ }^{4}$ We omit the dependence on $\mu$, which from now on is a fixed number.
} 
For $r \geq 1$, we will define $\mathfrak{g}_{n, r}=G_{F} / G_{F}^{r+1}$. For more details, see [32, Example 4, page 256 .

Definition 4.2. Denote by $n_{m, s}$ the dimension (as a vector space) of the free nilpotent Lie algebra $\mathfrak{g}_{m, s}$. Let $X_{1}, \ldots, X_{m}$ be a set of smooth vector fields defined in an open neighborhood of a point $x_{o} \in \mathbb{R}^{n}$, and let $n_{s}$ be the dimension of the space generated by all commutators of the $X_{j}$ 's of length $\leq s$ evaluated at the point $x_{o}$. We shall say that $X_{1}, \ldots, X_{m}$ are free up to step $r$ if for any $1 \leq s \leq r$ we have $n_{m, s}=n_{s}$.

Remark 4.3. We observe that if the vector fields $X_{1}, \ldots, X_{m}$ are free up to step $r$ in an open set $\Omega \subset \mathbb{R}^{n}$, then commutators of different lengths are linearly independent, while commutators of the same length may be linearly dependent only because of antisymmetry, or because of the Jacobi identity. Consequently, any $n$-tuple $Y_{i_{1}}, \ldots, Y_{i_{n}}$ of commutators which is a basis for $\mathbb{R}^{n}$ must have the same cumulative degree

$$
Q=\sum_{k=1}^{n} d_{i_{k}}=\sum_{j=1}^{r} j\left(n_{m, j}-n_{m, j-1}\right) .
$$

From this point on, we will denote by $Y_{1}, \ldots, Y_{m}$ the generators of the Lie algebra $\mathfrak{g}_{m, s}$.

Consider smooth vector fields $X_{1}, \ldots, X_{m}$ in $\mathbb{R}^{n}$ which are free up to step $r$ in the open set $\Omega \subset \mathbb{R}^{n}$, and let $\xi \in \Omega$. For each $k \in \mathbb{N}, 1 \leq k \leq r$, choose $\left\{X_{i k}\right\}$, commutators of length $k$ with $X_{i 1}=X_{i}$, such that the system $\left\{X_{i k}\right\}$, $k=1, \ldots, r$, evaluated at $\xi$ is a basis of $\mathbb{R}^{n}$. Then we can define a system of coordinates (canonical coordinates) associated to $\left\{X_{i k}\right\}$, based at the point $\xi$, as follows:

$$
\left(u_{j k}\right) \leftrightarrow \exp \left(\sum u_{j k} X_{j k}\right) \cdot \xi
$$

where $\exp (\cdot) \cdot \xi: T_{\xi} \mathbb{R}^{n} \rightarrow \mathbb{R}^{n}$ denotes the exponential map based at $\xi$.

Remark 4.4. Let us fix a collection of commutators $\left\{X_{i k}\right\}$ generating $\mathbb{R}^{n}$ at a point $\xi$. We define the box-like sets which are given in canonical coordinates $\left(u_{i k}\right)$ by the expression

$$
\operatorname{Box}(\delta)=\left\{u_{i k} \in \mathbb{R}, k=1, \ldots, r,\left|u_{i k}\right| \leq \delta^{i}\right\} .
$$

By virtue of Theorems 1-7 in 31, we know that there exist $C, R_{o}>0$, and one particular collection $\left\{X_{i k}\right\}$, for which one has

$$
B_{C}\left(\xi, C^{-1} \delta\right) \subset \operatorname{Box}(\delta) \subset B_{C}(\xi, C \delta),
$$

for any $0<\delta<R_{o}$. Since we are considering vector fields which are free up to step $r$ at $\xi$, then all choices of the collection of commutators $\left\{X_{i k}\right\}$ give rise to equivalent sets of coordinates. Consequently we can state that for any compact set $K \subset \subset \Omega$, there exist constants $C_{1}, C_{2}>0$ such that

$$
\operatorname{Box}\left(C_{1} \delta\right) \subset B_{C}(\xi, \delta) \subset \operatorname{Box}\left(C_{2} \delta\right),
$$

for any $\xi \in K$ and $0<\delta<R_{o}$.

Following Rothschild and Stein 32, pg. 273, we want to approximate the free vector fields $X_{1}, \ldots, X_{m}$ with left-invariant vector fields $\left\{Y_{k}\right\}, k=1, \ldots, m$, generating the free nilpotent Lie algebra $\mathfrak{g}_{m, r}$. Let $\boldsymbol{G}_{m, r}$ denote the Lie group associated 
to $\mathfrak{g}_{m, r}$. For $k=1, \ldots, r$ and $i=1, \ldots, m_{k}$, denote by $Y_{i k}$ a basis of the space $V_{k}$ in the stratification $\mathfrak{g}_{m, r}=V_{1} \oplus \cdots \oplus V_{r}$, and by $y_{i, k}$ the corresponding exponential coordinates in the group $\boldsymbol{G}_{m, r}$. We let $Y_{i 1}=Y_{i}, i=1, \ldots, m_{1}$, denote the algebra generators. If $\alpha$ denotes the multi-index $\{i, k\}$, then its degree is defined to be $|\alpha|=k$.

Our argument will depend crucially upon the following fundamental result (see 32, Theorem 5, page 273)

Theorem 4.5. Let $X_{1}, \ldots, X_{m}$ be a system of smooth vector fields in $\mathbb{R}^{n}$ such that

(i) $X_{1}, \ldots, X_{m}$ and their commutators up to rank $r$ generate $\mathbb{R}^{n}$, and

(ii) $X_{1}, \ldots, X_{m}$ are free up to rank $r$ at $\xi \in \mathbb{R}^{n}$.

Then there exist a neighborhood $V$ of $\xi$ and a neighborhood $U$ of the identity in $\boldsymbol{G}_{m, r}$ such that:

(A) Let $\eta=\exp \left(\sum u_{j k} X_{j k}\right) \cdot \xi$, denote the canonical coordinate chart $\eta \rightarrow u_{j k}$ for $V$ centered at $\xi$. The map $\theta: V \times V \rightarrow U \subset \boldsymbol{G}_{m, r}$ defined by

$$
\theta_{\xi}(\eta)=\theta(\xi, \eta)=\exp \left(\sum u_{j k} Y_{j k}\right)
$$

is a diffeomorphism onto its image.

(B) In the coordinate system given by $\theta_{\xi}$ one can write

$$
X_{i}=Y_{i}+\mathcal{R}_{i}, \quad i=1, \ldots, m,
$$

where $\mathcal{R}_{i}$ is a vector field of local degree less than or equal to zero, depending smoothly on $\xi$, i.e., for any smooth $f$,

$$
X_{i}\left(f\left(\theta_{\xi}(\cdot)\right)\right)=\left(Y_{i} f+\mathcal{R}_{i} f\right)\left(\theta_{\xi}(\cdot)\right) .
$$

Let us recall that a differential operator on a Carnot group $\boldsymbol{G}$ has local degree less than or equal to zero if, after taking the Taylor expansion at the origin of its coefficients, each term so obtained is an homogeneous operator of degree less than or equal to zero. More explicitly, denote by $\left\{y_{\alpha}\right\}, \alpha=(i k)$, the exponential coordinates in $\boldsymbol{G}_{m, r}$ associated to the vector fields $Y_{i k}$. We say that the vector field $R_{i}$ has degree less than or equal to zero if for any $N \in \mathbb{N}$ and any multi-index $\alpha=$ $(i k)$ one can find a function $g_{\alpha, i, N} \in C^{\infty}(\boldsymbol{G})$, with growth $g_{\alpha, i, N}(y)=O\left(\|y\|^{N}\right)$, such that

$$
\mathcal{R}_{i}=\sum_{l=1}^{r} \sum_{|\alpha|=l}\left(p_{\alpha, i, N}(y) \partial_{y_{\alpha}}+g_{\alpha, i, N}(y) \partial_{y_{\alpha}}\right)
$$

in a neighborhood of the origin. In (4.6), the functions $p_{\alpha, i, N}(y)$ depend on $N$ and are homogeneous group polynomials (see [17]) of degree less than or equal to $N$ and greater than or equal to $|\alpha|$. The notation $\partial_{y_{\alpha}}$ indicates a first order derivative along one of the group coordinates whose formal degree is $|\alpha|$. In other words, modulo lower order terms, the operator $R_{i}$ has order less than or equal to $|\alpha|-\operatorname{deg}\left(p_{\alpha, i, N}\right) \leq|\alpha|-|\alpha|=0$.

In order to simplify the notation, we will write $\mathfrak{g}$ instead of $\mathfrak{g}_{m, r}$, and $\boldsymbol{G}$ instead of $\boldsymbol{G}_{m, r}$. We will assume $X_{1}, \ldots, X_{m}$ satisfy the hypothesis of Theorem 4.5, and consider canonical coordinates $\left\{x_{i, k}\right\}$ given by $\theta_{x_{0}}$ in a neighborhood $U$ of a point $x_{0} \in \Omega \subset \mathbb{R}^{n}$. We will denote by $\left\{x_{i, k}\right\}$ both the coordinates in $U$ and the coordinates in $\theta_{x_{0}}(U) \subset \boldsymbol{G}$. Since the exponential map exp $: \mathfrak{g} \rightarrow \boldsymbol{G}$ is a diffeomorphism, 
we will not distinguish between points in $\boldsymbol{G}$ and in $\mathfrak{g}$. When we write that a certain function $f$ is $C^{l}$, for some $l \in \mathbb{N}$, we intend that the Euclidean derivatives $\partial_{x_{j_{1}, k_{1}}} \partial_{x_{j_{2}, k_{2}}} \cdots \partial_{x_{j_{s}, k_{s}}} f, 1 \leq s \leq l$, are continuous in a neighborhood of $x_{0}$. Equivalently, the lift of $f$ to the free Lie algebra $\mathfrak{g}$, given by $f^{\prime}(x)=f\left(\theta_{x_{0}}^{-1}(x)\right)$, must have the same property with respect to differentiation along the exponential coordinates $x_{i, k}$ in a neighborhood of the origin.

The reason why we assume that the vector fields are free is that since we do not have Taylor polynomials in $\mathbb{R}^{n}$ for $f$ with respect to the vector fields $X_{i}$, we want to use the Taylor polynomials for the lift $f^{\prime}$. To do this we need to compare the derivatives $X_{i} f$ and $X_{i} X_{j} f$ at $x_{0}$ with the expressions $Y_{i} f$ and $Y_{i} Y_{j} f$ at the origin in $\mathfrak{g}$.

Regarding the first Taylor polynomial, Theorem 4.5 yields immediately

$$
\nabla_{0} f=\left(X_{1} f, \ldots, X_{m} f\right)\left(x_{0}\right)=\left(Y_{1} f^{\prime}, \ldots, Y_{m} f^{\prime}\right)(0) .
$$

In the following we will use the notation $Y f^{\prime}:=\left(Y_{1} f^{\prime}, \ldots, Y_{m} f^{\prime}\right)(0)$. To study the relation between the second Taylor polynomial of $f^{\prime}$ and $f$ we need Theorem 4.5 Since the $\mathcal{R}_{i}$ are of order less than or equal to zero, we expect that

$$
X_{i} X_{j} f\left(x_{0}\right)=Y_{i} Y_{j} f^{\prime}(0)+\mathcal{B}_{i j} f^{\prime}(0),
$$

where

$$
\mathcal{B}_{i j} f^{\prime}(0)=\left(\mathcal{R}_{i} Y_{j}+\mathcal{R}_{i} \mathcal{R}_{j}+Y_{i} \mathcal{R}_{j}\right) f^{\prime}(0)
$$

is an operator of order less than or equal to one. The expressions (4.8) and (4.9) lead us to the following lemma.

Lemma 4.6. If $f, g \in C^{2}(U)$ with $\nabla_{0} f\left(x_{0}\right)=\nabla_{0} g\left(x_{0}\right)$, then $\mathcal{B}_{i j} f^{\prime}(0)=\mathcal{B}_{i j} g^{\prime}(0)$, and:

(i) If $Y_{i} Y_{j} g^{\prime}(0)=Y_{i} Y_{j} f^{\prime}(0)$, then $X_{i} X_{j} g\left(x_{0}\right)=X_{i} X_{j} f\left(x_{0}\right)$.

(ii) If $X_{i} X_{j} g^{\prime}\left(x_{0}\right)=X_{i} X_{j} f^{\prime}\left(x_{0}\right)$, then $Y_{i} Y_{j} g(0)=Y_{i} Y_{j} f(0)$.

Proof. Since we are interested in the value of $Y_{i} Y_{j} f^{\prime}$ at the origin, we may ignore the higher order terms $g_{\alpha, i, N}$. In fact, provided we choose $N>2 r$ in (4.7), such higher order terms will vanish at the origin together with all their derivatives up to order $2 r$.

Modulo higher order terms we may write $\mathcal{R}_{i}=\sum_{\alpha} p_{\alpha, i} \partial_{x_{\alpha}}$, where the $p_{\alpha, i}$ are homogeneous polynomials of order $\alpha$ and $\alpha$ runs over all multi-indices $\alpha=(i, k)$ with $k=1, \ldots, r$ and $i=1, \ldots, m_{k}$. Since we are interested in the value of $Y_{i} Y_{j} f^{\prime}$ at the origin, we may ignore the higher order terms (they will vanish at the origin). Since $f, g \in C^{2}$ (recall, this is the Euclidean $C^{2}$ ), then all their derivatives are in $L^{\infty}$. Let us compute

$$
\begin{aligned}
\mathcal{B}_{i j} f^{\prime}(0) & =\left(\mathcal{R}_{i} Y_{j}+\mathcal{R}_{i} \mathcal{R}_{j}+Y_{i} \mathcal{R}_{j}\right) f^{\prime}(0) \\
& =\sum_{\alpha, \beta}\left(p_{\beta, i} \partial_{x_{\beta}}\left(Y_{j} f^{\prime}\right)+Y_{i}\left(p_{\alpha, j} \partial_{x_{\alpha}} f^{\prime}\right)+p_{\beta, i} \partial_{x_{\beta}}\left(p_{\alpha, j} \partial_{x_{\alpha}} f^{\prime}\right)(0)\right) .
\end{aligned}
$$

\footnotetext{
${ }^{5}$ We drop the index $N>2 r$, which is fixed from now on.
} 
This is a sum of terms of order $\beta, \alpha-1$ and $\alpha+\beta-1$ respectively. So when evaluated at the origin we are left with

$$
\mathcal{B}_{i j} f^{\prime}(0)=\sum_{l=1}^{m}\left(Y_{i} p_{(1, l), j}\right) Y_{l} f^{\prime}(0)=\sum_{l=1}^{m} b_{i j l} \partial_{x_{l, 1}} f^{\prime}(0) .
$$

Since the remainder $\mathcal{B}_{i j} f^{\prime}(0)$ is determined by the horizontal gradient of $f^{\prime}$ at the origin, the same argument applied to $g$ yields the conclusion of the lemma.

Because of the presence of the remainder terms $\mathcal{R}_{i}$ in Theorem 4.5] we need to strengthen our definition of viscosity solution.

Consider the $\infty$-Laplacian homogeneous equation

$$
\mathcal{L}_{\infty} u:=\sum_{i, j=1}^{m} X_{i} X_{j} u X_{i} u X_{j} u=0 .
$$

This is a fully non-linear, degenerate elliptic equation.

Definition 4.7. Let $\Omega \subset \mathbb{R}^{n}$ be an open set, and $X_{1}, \ldots, X_{m}$ free Hörmander vector fields in $\Omega$. A function $u \in C(\Omega)$ is a viscosity subsolution of (4.12) in $\Omega$ if for every $x_{0} \in \Omega$ and every $C^{2}$ function $\phi$ defined in a neighborhood $U$ of $x_{0}$ such that $u\left(x_{0}\right)=\phi\left(x_{0}\right)$ and $u(x) \leq \phi(x)$ for $x \in U$, we have $\mathcal{L}_{\infty} \phi(0) \geq 0$. We say that $u$ is a viscosity supersolution at $x_{0}$ if $-u$ is a viscosity subsolution at $x_{0}$, and finally, $u$ is a viscosity solution if it is both a subsolution and a supersolution in the viscosity sense.

Remark 4.8. The main difference between Definitions 1.2 and 4.7 is that in the latter the test function $\phi$ needs to be in $C^{2}$ (the Euclidean space $C^{2}$ ) in a neighborhood of $x_{0} \in \mathbb{R}^{n}$, and not only in $\Gamma^{2}$. The reason for this choice is that we will need to apply a second order differential operator (in the definition of $B_{i j}$, below) to the lift of $\phi$ to the Lie group.

We are now ready to state the main theorem of this section

Theorem 4.9 (Aronsson Euler equation in the free vector fields setting). Let $\Omega \subset$ $\mathbb{R}^{n}$ be an open set, and let $X_{1}, \ldots, X_{m}$ be a system of smooth vector fields in $\mathbb{R}^{n}$ which are free up to step $r \in \mathbb{N}$ and satisfy Hörmander's finite rank condition in a neighborhood of $\Omega$. If $u \in \Gamma^{1}(\Omega)$ is an absolutely minimizing Lipschitz extension with respect to the Carnot-Carathéodory metric associated to $X_{1}, \ldots, X_{m}$, then $u$ is a viscosity solution of the $\infty$-Laplacian equation (4.12) in $\Omega$.

Remark 4.10. The proof of Theorem 4.9 follows closely the argument used in proving Theorem 1.4. In particular, the final step is given by Lemma 3.1, which still holds for general systems of smooth vector fields, and for sufficiently smooth absolute minimizers $u$. The extra regularity hypothesis $u \in \Gamma^{1}$ is used only at this latter point, at the end of the proof of Lemma 3.1] where in this more general setting we will not be able to use group mollifiers. At this stage, it is not clear to us whether the results in [21], [18] and 8] make it possible to mollify our minimizer so that the $L^{\infty}$ norm of the horizontal gradient of the mollification is bounded by the $L^{\infty}$ norm of the horizontal gradient of the minimizer.

Proof of Theorem 4.9. The proof follows closely that of the main theorem, so we will only give a sketch of the main steps, indicating the points where a substantially different argument is required. 
Step 1: We argue by contradiction and assume that there exists $\phi \in C^{2}$ in a neighborhood of the point $x_{0}$ such that

$$
\begin{gathered}
u\left(x_{0}\right)=\phi\left(x_{0}\right), \text { and } u<\phi \text { in a neighborhood of } x_{0}, \\
\sum_{i, j=1}^{m} X_{i} X_{j} \phi\left(x_{0}\right) X_{i} \phi\left(x_{0}\right) X_{j} \phi\left(x_{0}\right)<0 .
\end{gathered}
$$

We fix a basis of $\mathbb{R}^{n}$ given by commutators of order up to $r \in \mathbb{N}$ of the vector fields $X_{1}, \ldots, X_{m}$ at the point $x_{0}$. Let us denote by $\left\{X_{i k}\right\}$ such a basis, with $X_{i 1}=X_{i}$, $i=1, \ldots, m$. We use the exponential coordinates $x_{i, k}$ associated to this basis by

$$
\{x\}:=\exp \left(x_{i, k} X_{i k}\right) .
$$

By the Rothschild-Stein Theorem 4.5 we can associate to the system $X_{1}, \ldots, X_{m}$ a free nilpotent Lie algebra $\mathfrak{g}$ with $m$ generators and step $r \in \mathbb{N}$, and its corresponding Lie group $\boldsymbol{G}$. Let us denote by $Y_{i k}$ a basis of the Lie algebra. The same set of coordinates $\{x\}$ used in a neighborhood of $x_{0} \in \mathbb{R}^{n}$ can be used in a neighborhood of the origin on the Lie algebra $\mathfrak{g}$ and on the group $\boldsymbol{G}$, by means of the osculating map

$$
\theta_{x_{0}}(x)=\exp _{G}\left(x_{i, k} Y_{i k}\right) .
$$

As we did previously, we will again denote by $x_{1}=\left\{x_{1,1}, \ldots, x_{m, 1}\right\}$ and $x_{2}=$ $\left\{x_{1,2}, \ldots, x_{m_{2}, 2}\right\}$ the first two layers of the canonical coordinates.

Let $\phi^{\prime}(x)=\phi\left(\theta_{x_{0}}^{-1}(x)\right)$, and set

$$
\begin{gathered}
D^{\prime}=D=Y \phi^{\prime}(0), \\
T_{i}^{\prime}=Y_{i, 2} \phi^{\prime}(0) \text { and } T_{i}=X_{i, 2} \phi\left(x_{0}\right) \text { for } i=1, \ldots, m_{2}, \\
H_{i j}^{\prime}=\frac{1}{2}\left(Y_{i} Y_{j} \phi^{\prime}(0)+Y_{j} Y_{i} \phi^{\prime}(0)\right), \\
H_{i j}=\frac{1}{2}\left(X_{i} X_{j} \phi\left(x_{0}\right)+X_{j} X_{i} \phi\left(x_{0}\right)\right), \\
B_{i j}=\frac{1}{2}\left(\mathcal{B}_{i j} \phi^{\prime}(0)+\mathcal{B}_{j i} \phi^{\prime}(0)\right) .
\end{gathered}
$$

Notice that by virtue of (4.10) we have

$$
H^{\prime}+B=H .
$$

We will be interested in the second Taylor polynomial of $\phi^{\prime}$, which is given by

$$
P_{2}(x)=\left\langle D, y_{1}\right\rangle+\left\langle T^{\prime}, y_{2}\right\rangle+\frac{1}{2}\left\langle H^{\prime} y_{1}, y_{1}\right\rangle .
$$

Step 2: Arguing as in the proof of Theorem 1.4, we find a symmetric $m \times m$ matrix $M$ such that

$$
M>H^{\prime} \text { and } M D=(C-B) D,
$$

where $C$ is an $m \times m$ antisymmetric matrix given by

$$
C_{j i}=\frac{1}{2}\left[X_{i}, X_{j}\right] \phi\left(x_{0}\right) .
$$


In order to construct $M$ we will let $M=P+H^{\prime}$, where $P$ is a $m \times m$ symmetric matrix defined by the formula

$$
P z=z+\frac{\left\langle\left(C-B-H^{\prime}\right) D, z\right\rangle}{\langle(C-B-H) D, D\rangle}(C-B-H) D-\frac{\langle D, z\rangle}{\langle D, D\rangle} D,
$$

for any $z \in \mathbb{R}^{m}$. Since by (4.13) we have

$$
\begin{aligned}
\left\langle\left(C-B-H^{\prime}\right) D, D\right\rangle & =\left\langle\left(-B-H^{\prime}\right) D, D\right\rangle \\
& =\langle-H D, D\rangle>0,
\end{aligned}
$$

then it is easy to verify the following properties of $P$ : for every $z, z^{\prime} \in \mathbb{R}^{m}$,

$$
\begin{aligned}
& \langle P z, z\rangle>0, \\
& \left\langle P z, z^{\prime}\right\rangle=\left\langle P z^{\prime}, z\right\rangle, \\
& P D=\left(C-H^{\prime}-B\right) D \text {, hence }\langle P D, D\rangle=-\langle H D, D\rangle>0 .
\end{aligned}
$$

Step 3: Define a new $m \times m$ symmetric matrix $A$ given by $A=M+B$, and a plane $L=\left\{\left\langle H D, x_{1}\right\rangle=0\right\}$. Set $h(x)=\left\langle D, x_{1}\right\rangle+\left\langle T, x_{2}\right\rangle+\frac{1}{2}\left\langle M x_{1}, x_{1}\right\rangle$. Observe that

$$
\frac{1}{2}\left(\mathcal{B}_{i j} h^{\prime}(0)+\mathcal{B}_{j i} h^{\prime}(0)\right)=B_{i j} .
$$

Use the method of characteristics to find a smooth solution of the Cauchy problem for the horizontal eikonal equation

$$
\begin{cases}\left|\nabla_{0} V\right|=\left|\nabla_{0} \phi\left(x_{0}\right)\right| & \text { in a neighborhood } O \text { of } x_{0}, \\ V(x)=h(x) & \text { in } L \cap O,\end{cases}
$$

subject to the condition

$$
\nabla V\left(x_{0}\right)=\nabla \phi\left(x_{0}\right) \text { (the full Euclidean gradient) }
$$

Note that here we are using the fact that $\phi \in C^{1}$, since we are prescribing not only the horizontal derivatives, but also all the commutators evaluated at $x_{0}$.

If we let $\psi(x)=\left\langle H D, x_{1}\right\rangle$, then $X_{i} \psi\left(x_{0}\right)=\sum_{j=1}^{m} H_{i j} D_{j}$. The condition that $x_{0}$ is not characteristic for (4.18) is given by the inequality

$$
\sum_{i=1}^{m}\left(X_{i} V\right) X_{i} \psi\left(x_{0}\right)=\langle H D, D\rangle<0 .
$$

Step 4: We prove that

$$
\frac{1}{2}\left(X_{i} X_{j} V\left(x_{0}\right)+X_{j} X_{i} V\left(x_{0}\right)\right)=A_{i j} .
$$

In order to prove (4.21) we need to differentiate the equation (4.18) along the directions $X_{i}$ and evaluate the result at $x_{0}$, obtaining

$$
\sum_{j=1}^{m} X_{i} X_{j} V\left(x_{0}\right) X_{j} V\left(x_{0}\right)=0 \text {. }
$$


Consequently, we have

$$
\begin{aligned}
\sum_{j=1}^{m} \mathcal{V}_{i j} D_{j}:=\sum_{j=1}^{m} \frac{1}{2}\left(X_{i} X_{j} V\left(x_{0}\right)+X_{j} X_{i} V\left(x_{0}\right)\right) D_{j} & =\sum_{j=1}^{m} \frac{1}{2}\left[X_{j}, X_{i}\right] V\left(x_{0}\right) D_{j} \\
(\text { from } 4 \text { (4.19) }) & =\sum_{j=1}^{m} \frac{1}{2}\left[X_{j}, X_{i}\right] \phi\left(x_{0}\right) D_{j} \\
& =\sum_{j=1}^{m} C_{i j} D_{j} \\
\text { (from (4.14) }) & =\sum_{j=1}^{m} A_{i j} D_{j} .
\end{aligned}
$$

The latter gives us a control over the directions not in $L$. Now we turn our attention to the vectors in $L$. We observe that if $x \in L$, then the vector field $\sum_{i} x_{i, 1} X_{i}$ is tangent to $L$ at the point $x_{0}$. In fact,

$$
\frac{d}{d s}\left\langle H D, \exp \left(s \sum_{i} x_{i, 1} X_{i}\right)\right\rangle=\frac{d}{d s}\langle H D, s x\rangle=0 .
$$

Consider $x, y \in L$, and observe that since $h=V$ along $L$, then

$$
\sum_{i} x_{i, 1} X_{i}\left(\sum_{j} y_{j, 1} X_{j} V\right)\left(x_{0}\right)=\sum_{i} x_{i, 1} X_{i}\left(\sum_{j} y_{j, 1} X_{j} h\right)\left(x_{0}\right) .
$$

We now use (4.9) and compute

$$
\begin{aligned}
\left\langle\mathcal{V} x_{1}, y_{1}\right\rangle= & \left\langle\frac{1}{2}\left(Y_{i} Y_{j} h^{\prime}(0)+Y_{j} Y_{i} h^{\prime}(0)\right) x_{1}, y_{1}\right\rangle \\
& +\left\langle\frac{1}{2}\left(\mathcal{B}_{i j} h^{\prime}(0)+\mathcal{B}_{j i} h^{\prime}(0)\right) x_{1}, y_{1}\right\rangle \\
\text { from (4.17) }= & \left\langle M x_{1}, y_{1}\right\rangle+\left\langle B x_{1}, y_{1}\right\rangle=\left\langle A x_{1}, y_{1}\right\rangle .
\end{aligned}
$$

The rest of the proof follows along the lines of (3.24)-(3.26).

Step 5: We want to compare the second order Taylor polynomials $P_{2}$ and $P_{2}^{V^{\prime}}$ of (respectively) $\phi^{\prime}$ and $V^{\prime}(x)=V\left(\theta_{x_{0}}^{-1}(x)\right)$.

First of all, note that we have $P_{1}=P_{1}^{V^{\prime}}$, by virtue of Theorem 4.5, In view of Lemma 4.6 we also obtain

$$
\begin{aligned}
& \frac{1}{2}\left(Y_{i} Y_{j} V^{\prime}(0)+Y_{j} Y_{i} V^{\prime}(0)\right) \\
& \quad=\frac{1}{2}\left(X_{i} X_{j} V\left(x_{0}\right)+X_{j} X_{i} V\left(x_{0}\right)\right)-\frac{1}{2}\left(\mathcal{B}_{i j} V^{\prime}(0)+\mathcal{B}_{j i} V^{\prime}(0)\right) \\
& \quad=\frac{1}{2}\left(X_{i} X_{j} V\left(x_{0}\right)+X_{j} X_{i} V\left(x_{0}\right)\right)-\frac{1}{2}\left(\mathcal{B}_{i j} \phi^{\prime}(0)+\mathcal{B}_{j i} \phi^{\prime}(0)\right) \\
& \quad=A_{i j}-B_{i j} \\
& \quad=M_{i j}
\end{aligned}
$$

Since by (4.14) we have $M>H^{\prime}$, it follows that

$$
P_{2}^{V^{\prime}}(x)>P_{2}(x)
$$


for $x \neq 0$ and $x$ in a neighborhood of the origin (the two polynomials are equal at the origin).

The stratified Taylor inequality of Theorem 2.3 immediately yields

$$
V^{\prime}>\phi^{\prime}
$$

in $O \backslash\{0\}$, where $O$ is a neighborhood of the origin.

Arguing as in Theorem 1.4 we have

$$
u^{\prime}(x)<V_{e}^{\prime}(x):=V^{\prime}(x)-\epsilon,
$$

for $R \geq \sqrt{\frac{\epsilon}{\mu}}$ and $x \in \partial B(0, R)$.

At this point we want to rephrase the latter inequality in terms of the functions $u$ and $V$ near $x_{0}$. To do this, we need to understand what is the image of $B(0, R)$ under the action of the map $\theta_{x_{0}}^{-1}$.

We recall that by Remark 4.4 there exist $C, r_{0}>0$ such that for $0<\delta<r_{0}$,

$$
\operatorname{Box}\left(C^{-1} \delta\right) \subset B_{C}\left(x_{0}, \delta\right) \subset \operatorname{Box}(C \delta) .
$$

On the other hand, the box sets

$$
\left\{x \in \boldsymbol{G} \text { such that } \sum_{i=1}^{m_{k}} x_{i, k}^{2}<R^{2 k}, \text { for } k=1, \ldots, r\right\} \subset \boldsymbol{G}
$$

are equivalent to the pseudo-balls $B(0, R)$ in the group (this can be verified through elementary computations, for a reference see 31). Hence, there exist constants $C_{1}, r_{1}$ such that for $0<\delta<r_{1}$ one has

$$
\theta_{x_{0}}^{-1}\left(B\left(0, C^{-1} \delta\right)\right) \subset B_{C}\left(x_{0}, \delta\right) \subset \theta_{x_{0}}^{-1}(B(0, C \delta)) .
$$

By (4.26) and (4.27) we can find a neighborhood $W$ of $x_{0}$ such that for $R>\sqrt{\frac{\epsilon}{\mu}}$, $\epsilon$ small enough, and $x \in \partial B_{C}\left(x_{0}, C R\right) \cap W$, one has

$$
u(x)<V_{\epsilon}(x):=V(x)-\epsilon .
$$

On the other hand,

$$
u(0)=0>-\epsilon=V_{\epsilon}(0) .
$$

At this point we argue as in (3.28) and (3.29), and use Lemma 3.1 to reach a contradiction and conclude the proof of the theorem.

\section{A Class of VECTOR FIELD EXAMPLE}

In this section, we examine certain vector fields without a group structure and use the explicitly computed Taylor series to show that absolute minimizers are infinite harmonic. We begin by constructing the tangent space. Consider $\mathbb{R}^{3}$ with coordinates $\left(x_{1}, x_{2}, x_{3}\right)$ spanned by the vector fields

$$
\begin{aligned}
& X_{1}=\cos \left(x_{3}+\alpha\right) \frac{\partial}{\partial x_{1}}+\sin \left(x_{3}+\alpha\right) \frac{\partial}{\partial x_{2}}, \\
& X_{2}=\frac{\partial}{\partial x_{3}}, \\
& X_{3}=\sin \left(x_{3}+\alpha\right) \frac{\partial}{\partial x_{1}}-\cos \left(x_{3}+\alpha\right) \frac{\partial}{\partial x_{2}},
\end{aligned}
$$

where $\alpha$ is an arbitrary real number. Observe that since

$$
\cos ^{2}\left(x_{3}+\alpha\right)+\sin ^{2}\left(x_{3}+\alpha\right)=1,
$$


these vectors do indeed span $\mathbb{R}^{3}$; and since $\left[X_{1}, X_{2}\right]=X_{3}, X_{1}$ and $X_{2}$ satisfy Hörmander's condition. Note, though, that $X_{3}$ is therefore a second order derivative. We endow $\mathbb{R}^{3}$ with an inner product $\langle$,$\rangle so that the vectors X_{1}, X_{2}$ and $X_{3}$ are orthonormal, and we call the resulting manifold $g$, which will be the tangent space to a space denoted $G$, also using coordinates $x=\left(x_{1}, x_{2}, x_{3}\right)$ for points $x \in G$. We denote fixed points by $x^{0}=\left(x_{1}^{0}, x_{2}^{0}, x_{3}^{0}\right)$.

To assist in showing that $G$ is not a group, we state Proposition 4.10 from [4].

Proposition 5.1. Let $s$ be a non-negative integer and let $p \in G$. For a smooth function $f$ defined near $p$, the following are equivalent:

(1) $f(q)=O\left(d_{C}(p, q)^{s}\right)$ for $q$ near $p$.

(2) The [subelliptic] derivatives of order $\leq s-1$ of $f$ vanish at $p$.

The order of $f$ at $p$ is then the number $s_{0}$, so either of these conditions hold with $s_{0}$ but not with $s_{0}+1$. In particular, it is easy to see using the second condition that $x_{1}$ has order 1 unless $\cos \left(x_{3}+\alpha\right)=0$, in which case $x_{1}$ has order 2. Similarly, $x_{2}$ has order 1 , unless $\sin \left(x_{3}+\alpha\right)=0$, in which case $x_{2}$ has order 2 . Because group invariance implies all points have the same order, $G$ cannot be a group. Using the first condition, one then concludes that the Carnot-Carathéodory metric has different local estimates when based at different points. Fixing a point $x^{0}$, we obtain the local estimate at $x^{0}$ :

$$
d_{C}\left(x^{0}, x\right) \sim\left|x_{1}-x_{1}^{0}\right|^{a\left(x_{3}^{0}+\alpha\right)}+\left|x_{2}-x_{2}^{0}\right|^{b\left(x_{3}^{0}+\alpha\right)}+\left|x_{3}-x_{3}^{0}\right|,
$$

where the function $a(\theta)$ is defined by

$$
a(\theta)= \begin{cases}\frac{1}{2} & \text { if } \cos (\theta)=0 \\ 1 & \text { if } \cos (\theta) \neq 0\end{cases}
$$

and $b(\theta)$ is defined analogously using sin. Using the language of [4, one sees that our coordinates $\left(x_{1}, x_{2}, x_{3}\right)$ form a system of privileged coordinates (see p. 42 in [4]), and so equation (5.1) is merely a restatement of Theorem 7.34 in [4].

The change in order at various points not only reiterates that $G$ is not a group, but it also must be reflected in the Taylor polynomial. The surprising fact is that the Taylor polynomial based at an arbitrary point, given by the proposition below, can be expressed in a form independent of the order of $x_{1}$ and $x_{2}$. This fact is a consequence of the previous paragraph. In particular, we have the following proposition.

Proposition 5.2. Given a point $x^{0}=\left(x_{1}^{0}, x_{2}^{0}, x_{3}^{0}\right)$ and a $\Gamma^{2}$ function $u: G \mapsto \mathbb{R}$, for points $x$ near $x^{0}$ the second order Taylor polynomial is given by

$$
\begin{aligned}
u(x)= & u\left(x^{0}\right)+\left(x_{1}-x_{1}^{0}\right)\left(\cos \left(x_{3}+\alpha\right) X_{1} u\left(x^{0}\right)+\sin \left(x_{3}+\alpha\right) X_{3} u\left(x^{0}\right)\right) \\
& +\left(x_{2}-x_{2}^{0}\right)\left(\sin \left(x_{3}+\alpha\right) X_{1} u\left(x^{0}\right)-\cos \left(x_{3}+\alpha\right) X_{3} u\left(x^{0}\right)\right) \\
& +\left(x_{3}-x_{3}^{0}\right) X_{2} u\left(x^{0}\right)+\frac{1}{2}\left(x_{1}-x_{1}^{0}\right)^{2} X_{1} X_{1} u\left(x^{0}\right)+\frac{1}{2}\left(x_{2}-x_{2}^{0}\right)^{2} X_{1} X_{1} u\left(x^{0}\right) \\
& +\frac{1}{2}\left(x_{3}-x_{3}^{0}\right)^{2} X_{2} X_{2} u\left(x^{0}\right)+\cos \left(x_{3}+\alpha\right)\left(x_{1}-x_{1}^{0}\right)\left(x_{3}-x_{3}^{0}\right) X_{1} X_{2} u\left(x^{0}\right) \\
& +\sin \left(x_{3}+\alpha\right)\left(x_{2}-x_{2}^{0}\right)\left(x_{3}-x_{3}^{0}\right) X_{1} X_{2} u\left(x^{0}\right)+O\left(d_{C}\left(x, x^{0}\right)^{3}\right) .
\end{aligned}
$$


Note that this Taylor formula is valid for all points, including those at which $\cos \left(x_{3}+\alpha\right)$ or $\sin \left(x_{3}+\alpha\right)$ is 0 .

Proof. Denote the right hand side (without the error term) by $P(x)$. We then have

$$
\begin{aligned}
X_{1} P(x)= & \cos \left(x_{3}+\alpha\right)\left(\cos \left(x_{3}+\alpha\right) X_{1} u\left(x^{0}\right)+\sin \left(x_{3}+\alpha\right) X_{3} u\left(x^{0}\right)\right. \\
& \left.+\left(x_{1}-x_{1}^{0}\right) X_{1} X_{1} u\left(x^{0}\right)+\cos \left(x_{3}+\alpha\right)\left(x_{3}-x_{3}^{0}\right) X_{1} X_{2} u\left(x^{0}\right)\right) \\
& +\sin \left(x_{3}+\alpha\right)\left(\sin \left(x_{3}+\alpha\right) X_{1} u\left(x^{0}\right)-\cos \left(x_{3}+\alpha\right) X_{3} u\left(x^{0}\right)\right. \\
& \left.+\left(x_{2}-x_{2}^{0}\right) X_{1} X_{1} u\left(x^{0}\right)+\sin \left(x_{3}+\alpha\right)\left(x_{3}-x_{3}^{0}\right) X_{1} X_{2} u\left(x^{0}\right)\right) \\
= & X_{1} u\left(x^{0}\right)+\cos \left(x_{3}+\alpha\right)\left(x_{1}-x_{1}^{0}\right) X_{1} X_{1} u\left(x^{0}\right) \\
& +\sin \left(x_{3}+\alpha\right)\left(x_{2}-x_{2}^{0}\right) X_{1} X_{1} u\left(x^{0}\right)+\left(x_{3}-x_{3}^{0}\right) X_{1} X_{2} u\left(x^{0}\right)
\end{aligned}
$$

and

$$
\begin{aligned}
X_{2} P(x)= & X_{2} u\left(x^{0}\right)-\sin \left(x_{3}+\alpha\right)\left(x_{1}-x_{1}^{0}\right)\left(x_{3}-x_{3}^{0}\right) X_{1} X_{2} u\left(x^{0}\right) \\
& +\left(x_{3}-x_{3}^{0}\right) X_{2} X_{2} u\left(x^{0}\right)+\cos \left(x_{3}+\alpha\right)\left(x_{2}-x_{2}^{0}\right)\left(x_{3}-x_{3}^{0}\right) X_{1} X_{2} u\left(x^{0}\right) \\
& +\cos \left(x_{3}+\alpha\right)\left(x_{1}-x_{1}^{0}\right) X_{1} X_{2} u\left(x^{0}\right) \\
& +\sin \left(x_{3}+\alpha\right)\left(x_{2}-x_{2}^{0}\right) X_{1} X_{2} u\left(x^{0}\right)
\end{aligned}
$$

The second order derivatives can then be computed, producing

$$
\begin{aligned}
X_{3} P(x)= & \sin \left(x_{3}+\alpha\right)\left(\cos \left(x_{3}+\alpha\right) X_{1} u\left(x^{0}\right)+\sin \left(x_{3}+\alpha\right) X_{3} u\left(x^{0}\right)\right. \\
+ & \left.\left(x_{1}-x_{1}^{0}\right) X_{1} X_{1} u\left(x^{0}\right)+\cos \left(x_{3}+\alpha\right)\left(x_{3}-x_{3}^{0}\right) X_{1} X_{2} u\left(x^{0}\right)\right) \\
- & \cos \left(x_{3}+\alpha\right)\left(\sin \left(x_{3}+\alpha\right) X_{1} u\left(x^{0}\right)-\cos \left(x_{3}+\alpha\right) X_{3} u\left(x^{0}\right)\right. \\
+ & \left.\left(x_{2}-x_{2}^{0}\right) X_{1} X_{1} u\left(x^{0}\right)+\sin \left(x_{3}+\alpha\right)\left(x_{3}-x_{3}^{0}\right) X_{1} X_{2} u\left(x^{0}\right)\right) \\
=\quad X_{3} u\left(x^{0}\right)-\cos \left(x_{3}+\alpha\right)\left(x_{2}-x_{2}^{0}\right) X_{1} X_{1} u\left(x^{0}\right) & +\sin \left(x_{3}+\alpha\right)\left(x_{1}-x_{1}^{0}\right) X_{1} X_{1} u\left(x^{0}\right), \\
X_{1} X_{1} P(x)= & \cos ^{2}\left(x_{3}+\alpha\right) X_{1} X_{1} u\left(x^{0}\right)+\sin ^{2}\left(x_{3}+\alpha\right) X_{1} X_{1} u\left(x^{0}\right), \\
X_{2} X_{2} P(x)= & X_{2} X_{2} u\left(x^{0}\right)-\sin \left(x_{3}+\alpha\right)\left(x_{1}-x_{1}^{0}\right) X_{1} X_{2} u\left(x^{0}\right) \\
& +\cos \left(x_{3}+\alpha\right)\left(x_{2}-x_{2}^{0}\right) X_{1} X_{2} u\left(x^{0}\right) \\
& -\sin \left(x_{3}+\alpha\right)\left(x_{1}-x_{1}^{0}\right) X_{1} X_{2} u\left(x^{0}\right) \\
& -\cos \left(x_{3}+\alpha\right)\left(x_{1}-x_{1}^{0}\right)\left(x_{3}-x_{3}^{0}\right) X_{1} X_{2} u\left(x^{0}\right) \\
& +\cos \left(x_{3}+\alpha\right)\left(x_{2}-x_{2}^{0}\right) X_{1} X_{2} u\left(x^{0}\right) \\
& -\sin \left(x_{3}+\alpha\right)\left(x_{2}-x_{2}^{0}\right)\left(x_{3}-x_{3}^{0}\right) X_{1} X_{2} u\left(x^{0}\right), \\
= & \cos { }^{2}\left(x_{3}+\alpha\right) X_{1} X_{2} u\left(x^{0}\right)+\sin ^{2}\left(x_{3}+\alpha\right) X_{1} X_{2} u\left(x^{0}\right) .
\end{aligned}
$$

Evaluation at $x=x^{0}$ and the fact that $\left[X_{1}, X_{2}\right]=X_{3}$ produce

$$
X_{i} P\left(x^{0}\right)=X_{i} u\left(x^{0}\right)
$$


and also

$$
X_{i} X_{j} P\left(x^{0}\right)=X_{i} X_{j} u\left(x^{0}\right) .
$$

Note that this calculation is independent of the value of $\sin \left(x_{3}+\alpha\right)$ and $\cos \left(x_{3}+\alpha\right)$. Whenever $\sin \left(x_{3}+\alpha\right)=0, x_{2}$ is of order 2 , so that the term

$$
\sin \left(x_{3}+\alpha\right)\left(x_{2}-x_{2}^{0}\right)\left(x_{3}-x_{3}^{0}\right) X_{1} X_{2} u\left(x^{0}\right)
$$

is zero and does not appear in the Taylor polynomial, reflecting the fact that

$$
\left(x_{2}-x_{2}^{0}\right)\left(x_{3}-x_{3}^{0}\right)=O\left(d_{C}\left(x, x^{0}\right)^{3}\right) .
$$

A similar statement holds for $\cos \left(x_{3}+\alpha\right)$ and $\left(x_{1}-x_{1}^{0}\right)\left(x_{3}-x_{3}^{0}\right)$.

The error term follows from applying Proposition 5.1 to $P(x)-f(x)$.

Having established the second order Taylor polynomial, we wish to prove an analogue of Theorem 4.9 for the space $G$. We shall proceed with the proof, using the same five-step procedure to obtain a contradiction to Lemma 3.1

We first assume that we have a function $u \in \operatorname{Lip}(\Omega)$ that is an absolute minimizer but fails to be infinite harmonic at the origin. We will treat the general case later. Without loss of generality, we may assume that there is a $\Gamma^{2}$ function $\phi$ so that equations (3.13) hold. As in the previous proofs, we establish the following notation for matrices $H$ and $C$ and vectors $B$ and $D$ :

$$
\begin{aligned}
H_{i j} & =\frac{1}{2}\left(X_{i} X_{j} \phi(0)+X_{j} X_{i} \phi(0)\right), \\
D & =\nabla_{0} \phi(0), \\
B & =-H D, \\
C_{i j} & =\frac{1}{2}\left[X_{j}, X_{i}\right] \phi(0) .
\end{aligned}
$$

Step 1. We use equation (3.16) to define a matrix $P$ and the matrix $M=P+H$ with the properties of equation (3.17).

Step 2. We define the hyperplane $L$ by

$$
\begin{aligned}
& L=\left\{x_{1} X_{1}(0)+x_{2} X_{2}(0)+x_{3} X_{3}(0),\right. \\
& \left.\quad \text { with }\left(x_{1}, x_{2}, x_{3}\right) \in \mathbb{R}^{3} \text { such that } B_{1} x_{1}+B_{2} x_{2}=0\right\}
\end{aligned}
$$

and note that $\left(D, x_{3}\right) \notin L$ but $0 \in L$. We also define the $\Gamma^{2}$ function $h(x)$ by

$$
h(x)=P(x),
$$

where $P(x)$ is the polynomial from the proof of Proposition [5.2.

Step 3. We want to solve equation (3.18) (see also (4.18)) subject to the constraint $\nabla V(0)=\nabla \phi(0)$. Here $\nabla$ denotes the Euclidean gradient, i.e.,

$$
\begin{aligned}
\frac{\partial}{\partial x_{1}} V(0) & =\frac{\partial}{\partial x_{1}} \phi(0), \\
\frac{\partial}{\partial x_{2}} V(0) & =\frac{\partial}{\partial x_{2}} \phi(0), \\
\frac{\partial}{\partial x_{3}} V(0) & =\frac{\partial}{\partial x_{3}} \phi(0) .
\end{aligned}
$$

We also define the functions

$$
f(x, p)=\frac{1}{2}\left(\left(\cos \left(x_{3}+\alpha\right) p_{1}+\sin \left(x_{3}+\alpha\right) p_{2}\right)^{2}+\left(p_{3}\right)^{2}\right)
$$


and

$$
\psi(x)=\langle B, x\rangle .
$$

A simple computation shows that

$$
\nabla f(0, \nabla \phi(0))=\left(X_{1} \phi(0), X_{2} \phi(0)\right),
$$

and consequently

$$
\left\langle\nabla_{p} f(0, \nabla \phi(0)), \nabla_{x} \psi\right\rangle=\langle B, D\rangle \neq 0 .
$$

Interpreting the last inequality as the non-characteristic condition (compare with equation (3.20), we can argue as before and conclude the existence of $V$.

Step 4. Using calculations as in the Carnot group case, we obtain equation (3.21). We recall that the derivatives of $h(x)$ are computed in Proposition 5.2, so equation (3.25) is obtained by a straightforward computation.

Step 5. We replace $\|x\|$ in equation (3.27) by the norm

$$
\|x\|=\left|x_{1}-x_{1}^{0}\right|^{a\left(x_{3}^{0}+\alpha\right)}+\left|x_{2}-x_{2}^{0}\right|^{b\left(x_{3}^{0}+\alpha\right)}+\left|x_{3}-x_{3}^{0}\right|,
$$

with $a$ and $b$ defined as in equation (5.1). We then proceed as in the Carnot group case to reach a contradiction using Lemma 3.1.

Having completed the case when $u$ fails to be infinite harmonic at the origin, we next tackle the case when $u$ fails to be infinite harmonic at an arbitrary point $x^{0}$. Because $G$ is not a group, we do not have left translation, and in fact, the vector fields are not invariant. We cannot move from a point at which $x_{1}$ has order 1 to a point where it has order 2 . However, we can adjust the parameter $\alpha$ so that $x_{1}$ and $x_{2}$ have the same order at the origin as they do at $x^{0}$. In particular, at $x^{0}$ the vector fields $X_{1}, X_{2}$ and $X_{3}$ are given by

$$
\begin{aligned}
& X_{1}=\cos \left(x_{3}^{0}+\alpha\right) \frac{\partial}{\partial x_{1}}+\sin \left(x_{3}^{0}+\alpha\right) \frac{\partial}{\partial x_{2}}, \\
& X_{2}=\frac{\partial}{\partial x_{3}}, \\
& X_{3}=\sin \left(x_{3}^{0}+\alpha\right) \frac{\partial}{\partial x_{1}}-\cos \left(x_{3}^{0}+\alpha\right) \frac{\partial}{\partial x_{2}},
\end{aligned}
$$

and so at the origin we define the vector fields

$$
\begin{aligned}
Y_{1} & =\cos \beta \frac{\partial}{\partial x_{1}}+\sin \beta \frac{\partial}{\partial x_{2}} \\
Y_{2} & =\frac{\partial}{\partial x_{3}} \\
Y_{3} & =\sin \beta \frac{\partial}{\partial x_{1}}-\cos \beta \frac{\partial}{\partial x_{2}},
\end{aligned}
$$

choosing the parameter $\beta$ so that $x_{1}$ and $x_{2}$ have the same order at the origin with respect to the $Y$ vector fields as they do at $x^{0}$ with respect to the $X$ vector fields. We consider the function $\tilde{u}(x)=u\left(x+x^{0}\right)$, which is not infinite harmonic at the origin. In addition, elementary calculations show that Lip $\tilde{u}=\operatorname{Lip} u$ and $\tilde{u}$ is an absolute minimizer on $\tilde{\Omega} \equiv \Omega-x^{0}$. We then use the above proof to obtain a contradiction with Lemma 3.1 and conclude that $u$ is infinite harmonic at $x^{0}$. 
At this point, we note that upon completion of the case when $u$ is $\Gamma^{1}$, we observe that the lack of dilations in this setting does not allow us to remove the regularity assumption.

\section{REFERENCES}

[1] G. Aronsson, Extension of functions satisfying Lipschitz conditions, Ark. Mat. 6 (1967), 551-561. MR 36:754

[2] G. Aronsson, On the partial differential equation $u_{x}^{2} u_{x x}+2 u_{x} u_{y} u_{x y}+u_{y}^{2} u_{y y}$, Ark. Mat. 7 (1968), 395-425. MR 38:6239

[3] E. N. Barron, R. R. Jensen, and C. Y. Wang, The Euler equation and absolute minimizers of $L^{\infty}$ functionals, Arch. Rat. Mech. Anal. 157 (2001), 255-283. MR 2002m:49006

[4] A. Bellaïche, The tangent space in sub-Riemannian geometry. Sub-Riemannian geometry, Progr. Math., 144 (1996), Birkhäuser, 1-78. MR 98a:53108

[5] T. Bieske, On $\infty$-harmonic functions on the Heisenberg group, Comm. in PDE., 27, 3 and 4 (2002) 727-761. MR 2003g:35033

[6] T. Bieske, Viscosity solutions on Grushin-type planes, Illinois J. Math. 46 (2002), no. 3, 893-911. MR 2003k:35037

[7] T. Bieske, Lipschitz extensions on generalized Grushin-type spaces., preprint available at www.math.lsa.umich.edu/ ${ }^{\sim}$ tbieske.

[8] L. Capogna, D. Danielli and N. Garofalo, Subelliptic mollifiers and a characterization of Rellich and Poincarè domains. Partial differential equations, I (Turin, 1993). Rend. Sem. Mat. Univ. Politec. Torino 51 (1993), no. 4, 361-386. MR 96b:35030

[9] J. Cheeger, Differentiability of Lipschitz functions on metric measure spaces. Geom. Funct. Anal. 9 (1999), no. 3, 428-517. MR 2000g:53043

[10] W.L. Chow, Über System von linearen partiellen Differentialgleichungen erster Ordnug, Math. Ann., 117 (1939), 98-105. MR 1:313d

[11] M. G. Crandall, An efficient derivation of the Aronsson equation. Arch. Ration. Mech. Anal. 167 (2003), no. 4, 271-279. MR 2004b:35053

[12] M. G. Crandall, L. C. Evans and R. Gariepy, Optimal Lipschitz extensions and the infinity Laplacian, Calc. Var. Partial Differential Equations 13 (2001), no. 2, 123-139. MR 2002h:49048

[13] D. Danielli, N. Garofalo and D.M. Nhieu, Notions of convexity in Carnot groups, Comm. Anal. Geom. 11 (2003), 263-341. MR 2014879

[14] D. Danielli, N. Garofalo and D.M. Nhieu, On the best possible character of the $L^{Q}$ norm in some a-priori estimates for non-divergence form equations in Carnot groups, Proc. Amer. Math. Soc. 131 (2003), 3487-3498. MR 1991760

[15] L. C. Evans, Partial differential equations, Graduate studies in mathematics vol. 19, American Mathematical Society, Providence (1998). MR 99e:35001

[16] G. Folland, Subelliptic estimates and function spaces on nilpotent Lie groups, Ark. Mat., 13 (1975), 161-207. MR 58:13215

[17] G.B. Folland \& E.M. Stein, Hardy Spaces on Homogeneous Groups, Princeton Univ. Press., (1982). MR 84h:43027

[18] B. Franchi, R. Serapioni, and F. Serra Cassano, Meyers-Serrin type theorems and relaxation of variational integrals depending on vector fields. Houston J. Math. 22 (1996), no. 4, 859-890. MR 98c:49037

[19] N. Garofalo, Analysis and Geometry of Carnot-Carathéodory Spaces, With applications to $P D E$ 's, Birkhäuser, book in preparation.

[20] N. Garofalo \& D.M. Nhieu, Isoperimetric and Sobolev inequalities for Carnot-Carathéodory spaces and the existence of minimal surfaces, Comm. Pure Appl. Math., 49 (1996), 10811144. MR 97i:58032

[21] N. Garofalo \& D.M. Nhieu, Lipschitz continuity, global smooth approximations and extension theorems for Sobolev functions in Carnot-Carathéodory spaces. J. Anal. Math. 74 (1998), 6797. MR 2000i:46025

[22] M. Gromov, Carnot-Carathéodory spaces from within, in Subriemannian geometry, Progr. Math., 144 (1996), Birkhäuser, 79-323. MR 2000f:53034

[23] P. Hajlasz, Sobolev spaces on an arbitrary metric space. Potential Anal. 5 (1996), no. 4, 403-415. MR 97f:46050 
[24] J. Heinonen, Lectures on analysis on metric spaces Universitext. Springer-Verlag, New York, 2001. MR 2002c:30028

[25] L. Hörmander, Hypoelliptic second-order differential equations, Acta Math., 119 (1967), 147171. MR 36:5526

[26] R. Jensen, Uniqueness of Lipschitz extensions minimizing the sup-norm of the gradient, Arch. Rat. Mech. Anal. 123 (1993), 51-74. MR 94g:35063

[27] F. John, Partial differential equations (4th edition). Springer Verlag, New York (1991). MR 93f:35001

[28] P. Juutinen, Absolutely minimizing Lipschitz extensions in a metric space, Ann. Acad. Sci. Fenn. Math. 27 (2002), no. 1, 57-67. MR 2002m:54020

[29] G. Lu, J. Manfredi and B. Stroffolini, Convex functions on the Heisenberg group, to appear in Calc. Var. PDE.

[30] J. Mitchell, On Carnot-Carathéodory metrics. J. Differential Geom. 21 (1985), no. 1, 35-45. MR 87d:53086

[31] A. Nagel, E.M. Stein \& S. Wainger, Balls and metrics defined by vector fields. I: Basic properties, Acta Math., 155 (1985), 103-147. MR 86k:46049

[32] L. P. Rothschild \& E. M. Stein, Hypoelliptic differential operators and nilpotent groups. Acta Math. 137 (1976), 247-320. MR 55:9171

[33] E.M. Stein, Harmonic Analysis: Real Variable Methods, Orthogonality and Oscillatory Integrals, Princeton Univ. Press., (1993). MR 95c:42002

[34] C. Wang, The Euler equation of absolutely minimizing Lipschitz extensions for vector fields satisfying Hörmander's condition, preprint (2003).

Department of Mathematics, University of Michigan, Ann Arbor, Michigan 48109 E-mail address: tbieske@umich.edu

Current address: Department of Mathematics, University of South Florida, Tampa, Florida 33620

E-mail address: tbieske@math.usf.edu

Department of Mathematics, University of Arkansas, Fayetteville, Arkansas 72701

E-mail address: lcapogna@uark.edu 